\title{
FLOODS OF OCTOBER 1986 IN
}

\section{SOUTHCENTRAL ALASKA}

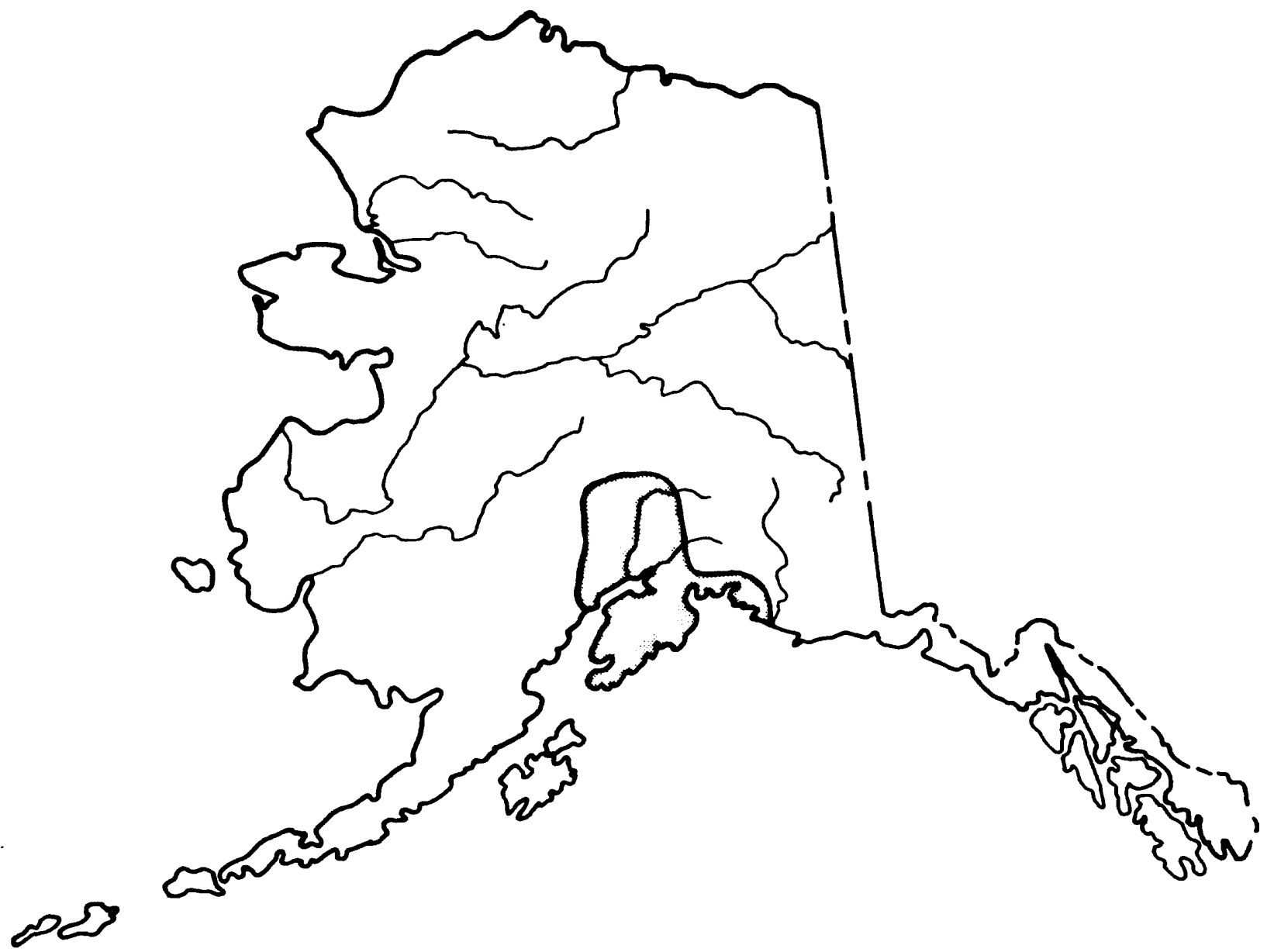

U.S. GEOLOGICAL SUR VEY

OPEN- FILE REPORT 87-391

REVISED 1988

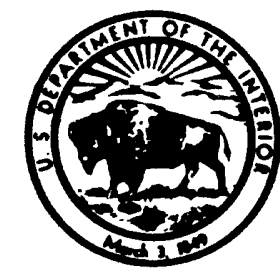

Prepared in cooperation with the:

ALASKA DEPARTMENT OF TRANSPORTATION AND PUBLIC FACILITIES ALASKA DIVISION OF EMERGENCY SERVICES FEDERAL HIGHWAY ADMINISTRATION 
FLOODS OF OCTOBER 1986 IN SOUTHCENTRAL ALASKA

by Robert D. Lamke and Bruce $\mathrm{B}$. Bigelow

U.S. GEOLOGICAL SURVEY

Open-File Report 87-391 REVISED 1988

Prepared in cooperation with the:

ALASKA DEPARTMENT OF TRANSPORTATION AND PUBLIC FACILITIES

ALASKA DIVISION OF EMERGENCY SERVICES

FEDERAL HIGHWAY ADMINISTRATION

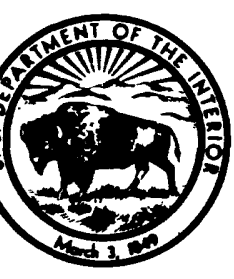

Anchorage, Alaska 
DEPARTMENT OF THE INTERIOR

DONALD PAUL HODEL, Secretary

U.S. GEOLOGICAL SURVEY

Dallas L. Peck, Director

For additional information write to:

District Chief

U.S. Geological Survey Water Resources Division 4230 University Drive, Suite 201 Anchorage, Alaska 99508-4664
Coples of this report can be purchased from:

U.S. Geological Survey Books and Open-File Reports Section Federal Center Box 25425

Denver, Colorado 80225 


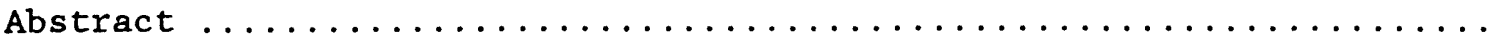

Introduction

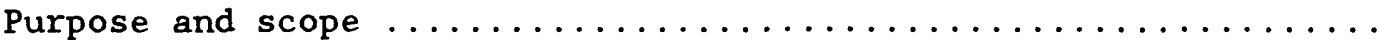

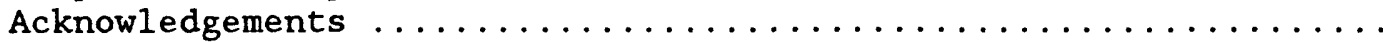

Precipitation

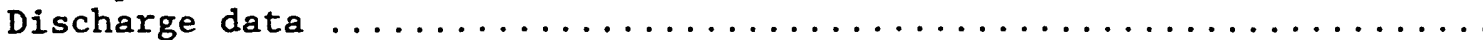

Peak stage and discharge table

Discharge data for October $1986^{\circ}$

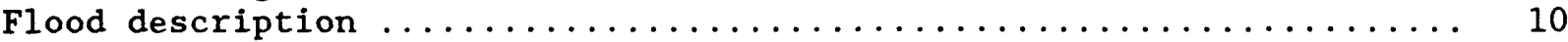

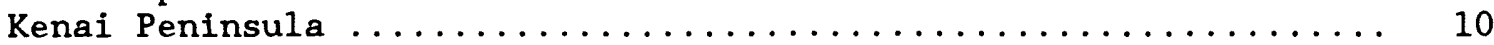

Bradley Lake and Homer area streams ................... 10

Seward area streams ............................. 14

Kenai Lake tributaries ......................... 17

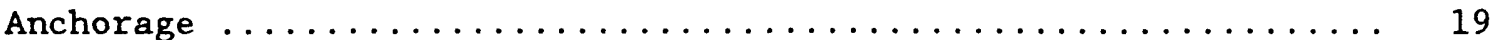

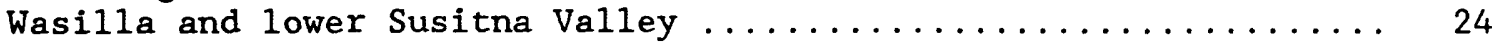

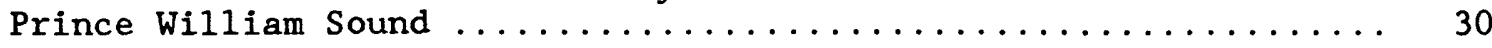

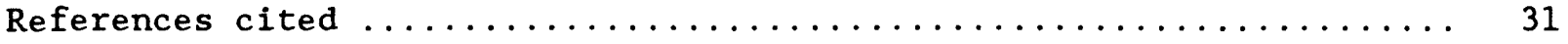

\section{ILLUSTRATIONS}

Figure 1. Map showing index to location of peak-discharge measurement sites for flood of October 10-12, 1986 in Southcentral

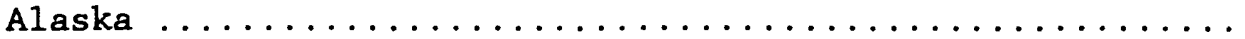

2. Map showing selected rainfall measurement sites 1 to 43 and isohyets of total precipitation, October 9-11, 1986, for

Southcentral Alaska ......................

3. Graphs showing accumulated precipitation at selected sites,

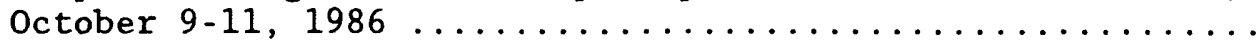

4. Map showing rainfall measurement sites 9 to 11 and peakdischarge measurement sites 20 to 28 , Bradley Lake to Homer.

5. Flood discharge hydrograph and concurrent 6-hour storm precipitation at selected sites in Kenai Peninsula ........

6. Map showing rainfall measurement sites 7 to 8,13 to 15 and peak-discharge measurement sites 6 to 19,29 to 37 , Seward

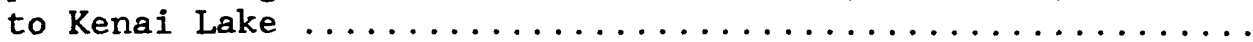

7. Cross sections at selected sites in Kenai Peninsula, showing channel changes after flood of October 11, $1986 \ldots$.

8. Map showing rainfall measurement sites 4,20 to 26,35 and peak-discharge measurement sites 4, 40 to 45 in Anchorage

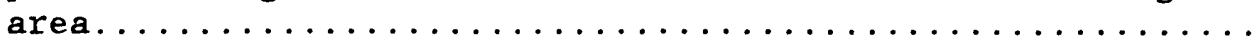

9. Flood discharge hydrographs, concurrent 6 -hour storm precipitation, and air temperatures at selected sites in

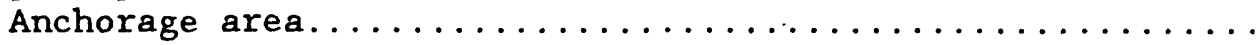

10. Map showing rainfall measurement sites 25 to 35,38 to 40 and peak-discharge measurement sites 46 to 51,54 to 65 , 67 to 68 in Wasilla and lower Susitna Valley area..........

11. Flood discharge hydrographs and concurrent 6-hour storm precipitation at selected sites in Susitna River basin ..... 
TABLES

Page

Table 1. Rainfall totals for October 9-11, 1986 storm in flood area ... 4

2. Summary of flood stages and discharges for the flood of

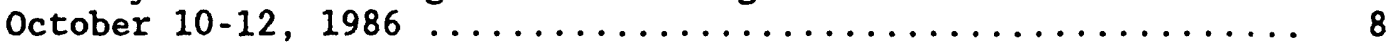

3. Discharge data for Upper Bradley River during October $1986 \ldots 12$

4. Discharge data for Campbell and East Fork Eklutna Creeks

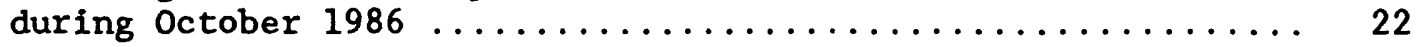

5. Discharge data for three selected gaging stations in lower Susitna River basin during October $1986 \ldots \ldots \ldots \ldots \ldots \ldots \ldots$

\section{CONVERSION TABLE}

For readers who may prefer to use metric (International system) units rather than inch-pound units, the conversion factors for the terms used in this report are listed below:

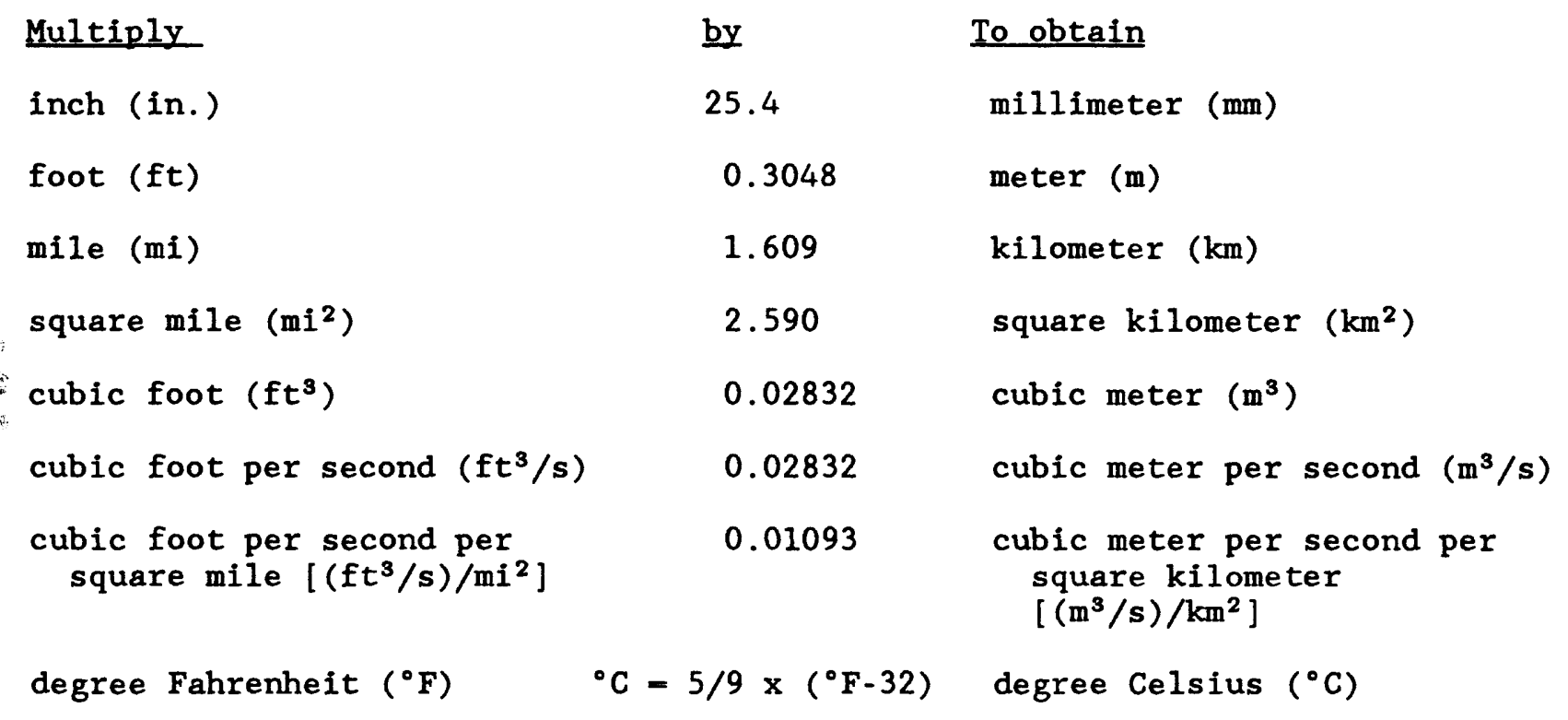

\section{Sea level:}

In this report "sea level" refers to the National Geodetic Vertical Datum of 1929 (NGVD of 1929)--a geodetic datum derived from a general adjustment of the first-order level nets of both the United States and Canada, formerly called "Mean Sea Level of 1929." 
FLOODS OF OCTOBER 1986 IN SOUTHCENTRAL ALASKA

by Robert D. Lamke and Bruce B. Bigelow

\begin{abstract}
Heavy precipitation associated with a large storm system resulted in major flooding in several areas of southcentral Alaska during October 10-12, 1986. Flooding was particularly severe in the Seward area of the Kenai Peninsula and in tributaries to Susitna River from Talkeetna downstream. Flood damage has been estimated at $\$ 20$ million and the region was declared a Federal disaster area.

The report includes a brief discussion of meteorological conditions that caused the unusual amounts of precipitation, a summary of flood stages and discharges, a comparison to prior floods, flood-frequency estimates, a brief description of flood areas, and hydrologic data for each area.
\end{abstract}

\title{
INTRODUCTION
}

Unusually large amounts of rainfall that began during mid-morning of October 9 and generally persisted until the evening of October 11 caused flooding in many areas in southcentral Alaska. Flooding generally began on the 10 th and most streams peaked on the 11th (with the exception of larger streams, which peaked later). The areas hardest hit by the flooding were near Seward on the Gulf of Alaska and the lower Susitna River Valley - - west of Talkeetna, southward from Talkeetna to Willow, and then southwest toward Tyonek. The most extensive damage was in Seward and along the Susitna River tributaries that cross the Parks Highway. Total damage has been estimated to be in excess of $\$ 20$ million; a large part of the damage was to the Alaska Highway system and the Alaska Railroad.

\section{Purpose and Scope}

This report presents a brief description of the floods, a summary of pertinent streamflow data, and estimated flood frequencies. Data on the magnitude and frequency of peak discharges and volumes of floodflows are useful to agencies involved in planning and design. The area covered in this report is shown in figure 1. The peak-discharge measurement sites shown in figure 1 (and more detailed maps of selected portions of the flood area) are in the downstream order system used by the U.S. Geological Survey. This revised report replaces the initial report of 1987 . It incorporates additional data not previously available; some of the discharge values, originally called provisional, have been verified and others were changed after additional review. 


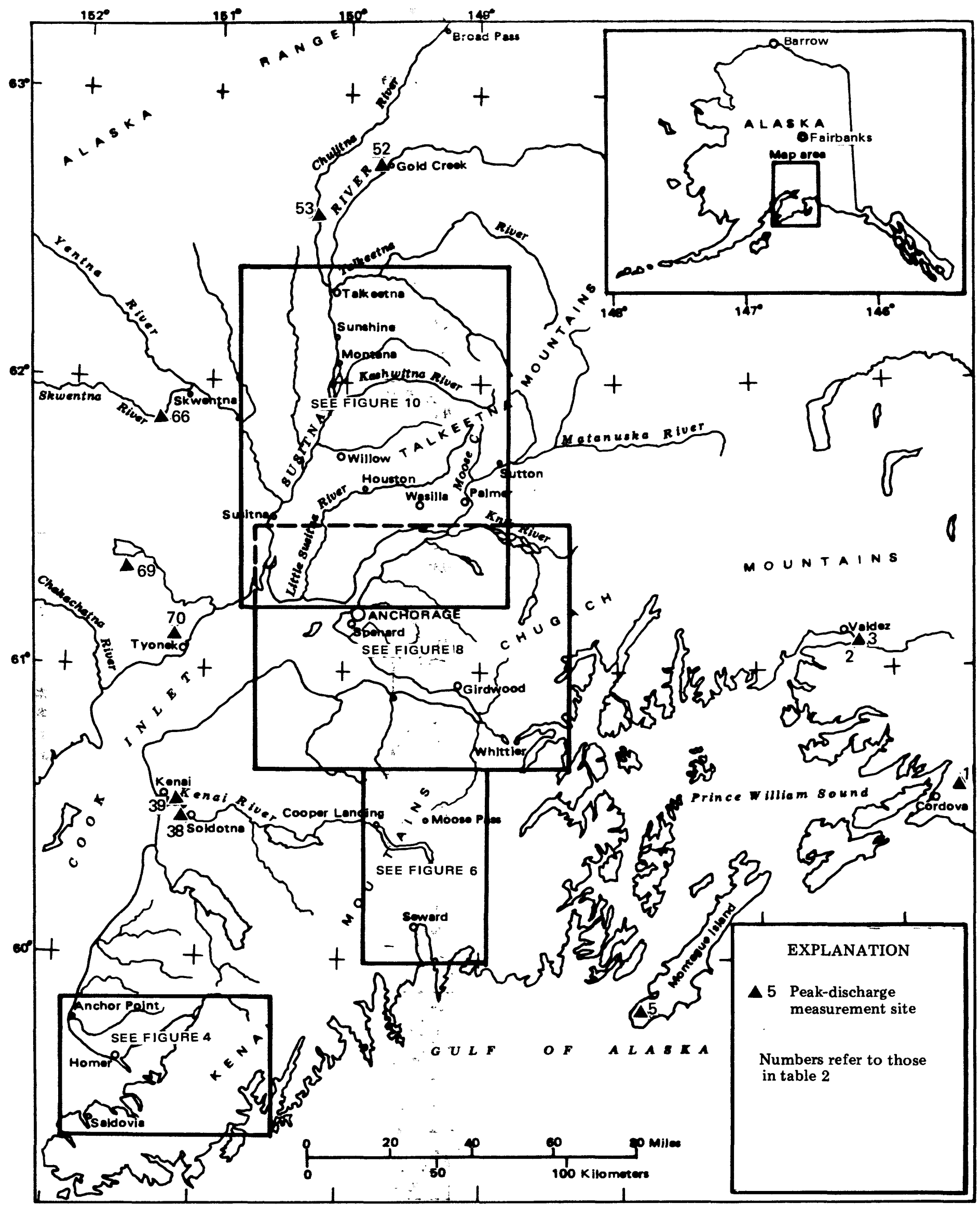

Figure 1. - Index to location of peak-discharge measurement sites for flood of October 10-12, 1986 in Southcentral Alaska. 


\section{Acknowledgements}

The data in this report were collected as part of the cooperative programs between the Geological Survey and various State of Alaska agencies: Department of Transportation and Public Facilities, Division of Geological and Geophysical Surveys of Department of Natural Resources, and Department of Fish and Game; and in cooperation with Kenai Peninsula Borough and the Municipality of Anchorage. Supplementary funding for the fieldwork and preparation of this report was furnished by the Alaska Division of Emergency Services, Alaska Department of Transportation and Public Facilities, and the Federal Highway Administration. Meteorological data were furnished by Gerald Nibler (1986) of the National Weather Service and an assessment of the flood was made by the Federal Emergency Management Agency (1986). The U.S. Forest Service and the U.S. Army Corps of Engineers each provide funds for two gaging stations used in the report.

\section{PRECIPITATION}

Precipitation totals for the 3-day storm period, October 9-11, are listed in table 1. An isohyetal map (fig. 2) shows that the rains were concentrated around Seward and in the lower Susitna River basin. Intense rainfall began first in the lower Kenai Peninsula and then moved eastward toward Seward. Precipitation data collected at Seward (fig. $3 \mathrm{~A}$ ) show that a period of heavy precipitation began the evening of October 9 and continued into the morning of October 11. The heaviest precipitation within a 6 -hour period occurred on October 10 from 4 to $10 \mathrm{a} . \mathrm{m}$., during which 6.00 in. of rain were recorded. The 24-hour period between October 10 at $4 \mathrm{a} . \mathrm{m}$. and October 11 at $4 \mathrm{a} . \mathrm{m}$. had 15.05 in. According to U.S. Weather Bureau data (1963), these rainfall totals have return periods of 50 years and of more than 100 years, respectively. Precipitation accumulations for two other stations on the Kenai Peninsula, Kenai and Lawing, are shown in figure 3B. The heavy rains around Seward were caused by a low-pressure trough oriented along $150^{\circ}$ west longitude that was blocked by a high-pressure ridge along the coast of British Columbia and southeast Alaska. The resultant storm front was stationary for 36 hours and the combined dynamic actions of the storm and orographic uplift produced a long period of intense precipitation around the Seward area. The system weakened and slowly shifted eastward to cause flood-producing rains near Cordova (Nibler, 1986) about 24 hours later. Accumulated precipitation at the Cordova Airport weather station is shown in figure 3D.

The unusually large precipitation in the vicinity of Talkeetna and Willow had an added factor in its cause (Nibler, 1986). A moisture-bearing jet in the lower atmosphere moved northward from the Kenai Peninsula, into upper Cook Inlet and Susitna Valley. During this time, a low-pressure trough over Barrow and an associated cold front moved southeastward across interior Alaska towards Fairbanks. When the two systems met, the circulation pattern of the trough was superimposed on the northward-moving jet of moist air and turned it eastward. This resulted in exceptionally heavy rains along the western slopes of the Talkeetna Mountains and in the Susitna Valley west of Talkeetna. The largest 6hour precipitation observed at the Talkeetna precipitation gage was 1.78 in. between $10 \mathrm{a} . \mathrm{m}$. and $4 \mathrm{p} . \mathrm{m}$. on October 10; the greatest 24-hour amount observed was $5.14 \mathrm{in.} \mathrm{from} 10 \mathrm{a} . \mathrm{m}$. on October 10 to $10 \mathrm{a.m}$. on October 11 . These values have return periods of 10 and 100 years, respectively (U.S. Weather Bureau, 
Table 1. - Rainfall totals for October 9-11, 1986 storm in flood area

[Station names and locations from U.S. Department of Comerce, 1986, except those footnoted below. Rainfall totals from Nibler, 1986 and U.S. Department of Comerce, 1986. Some sites are Nat1onal Weather Service observer stations read once dally; totals from most of these were adjusted to reflect period between midnights of October 8 and October 11.]

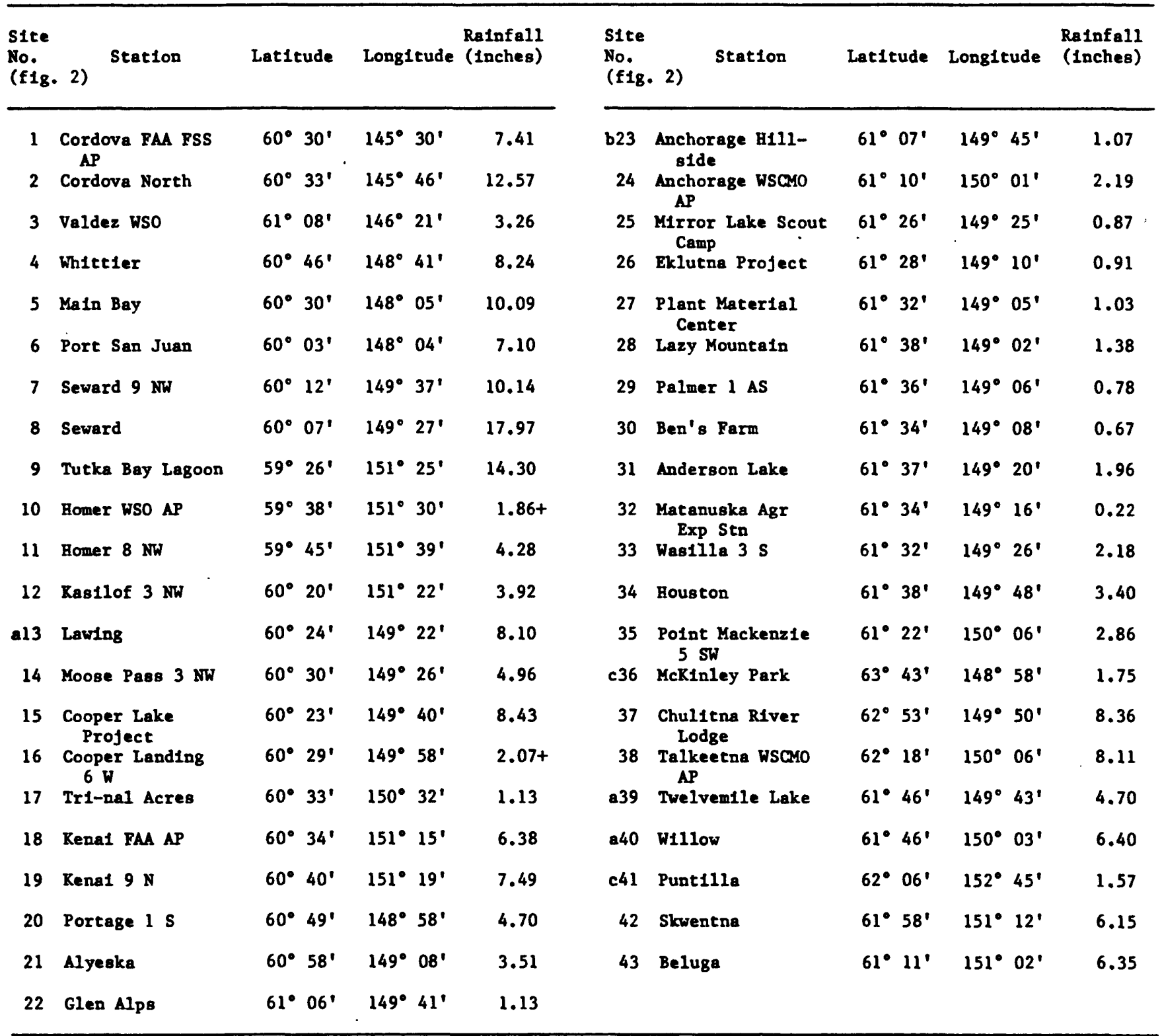

a Not official National Weather Service precipitation station; recording rain gage operated by another agency.

b Unofficial National Weather Service rain gage.

c Not on figure 2; outside map area. 


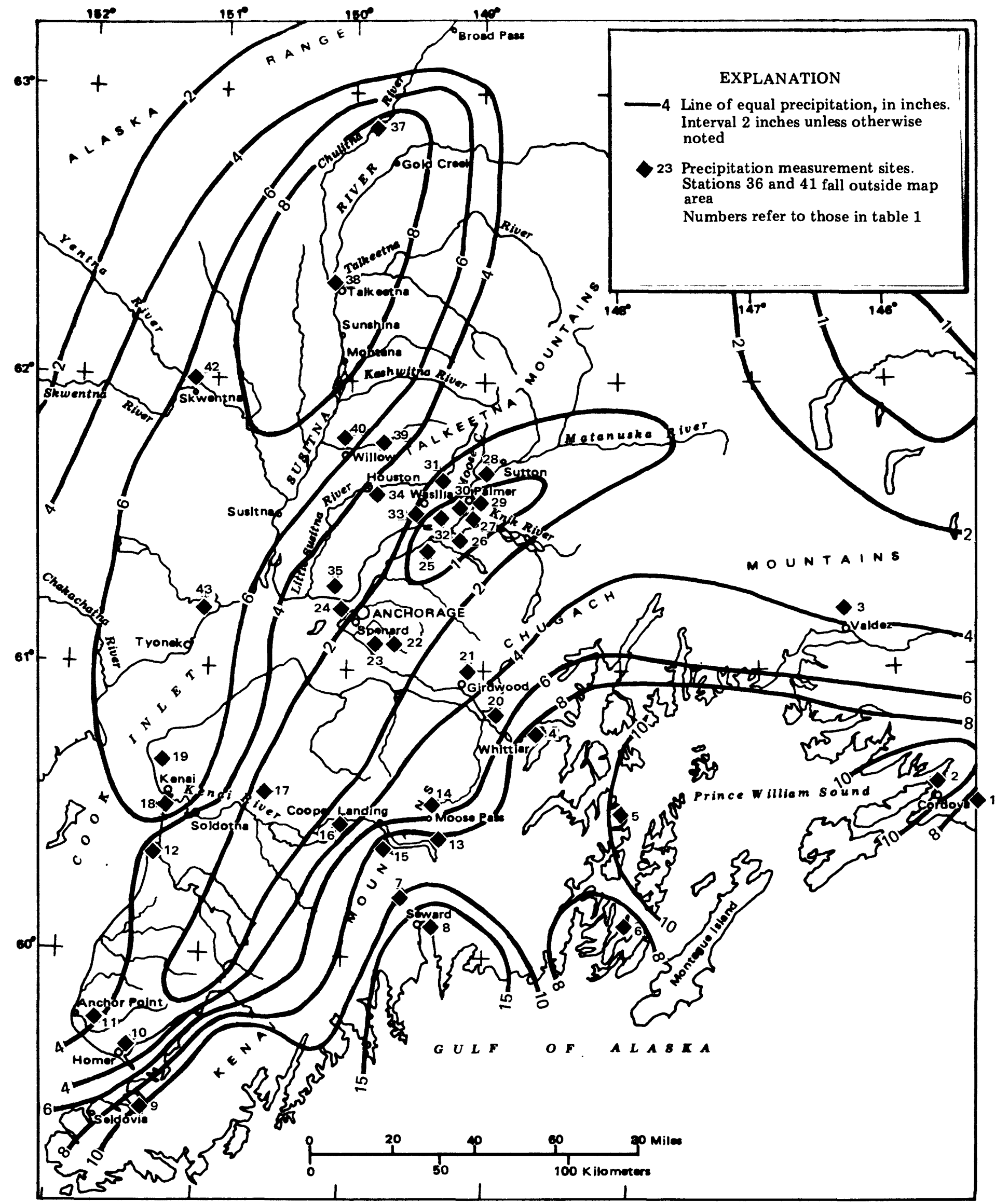

Figure 2.--Selected rainfall measurement sites 1 to 43 and isohyets of total precipitation, October 9-11, 1986, for Southcentral Alaska. Adapted from map furnished by National Weather Service. 


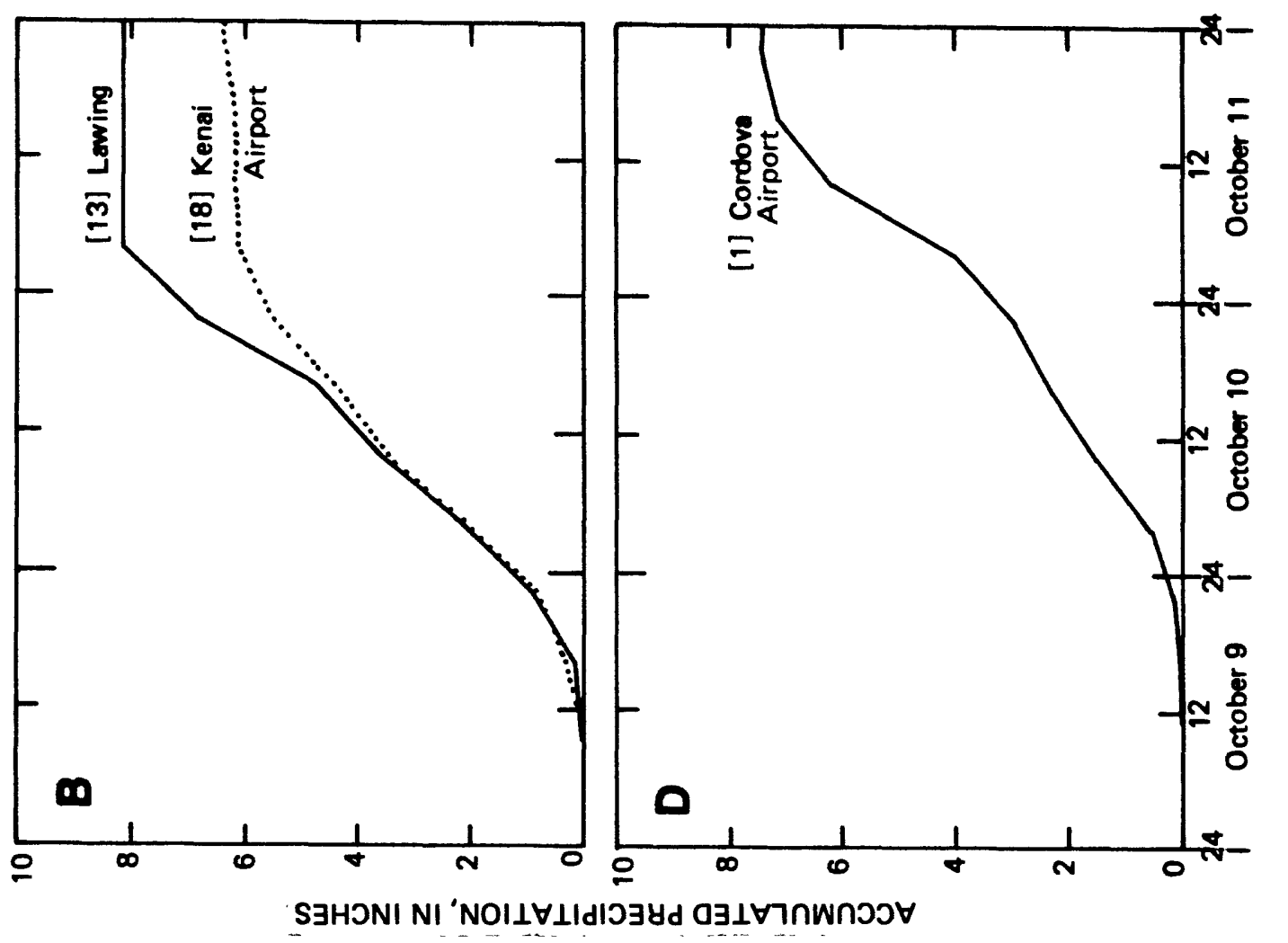

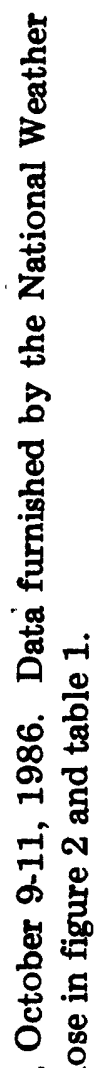

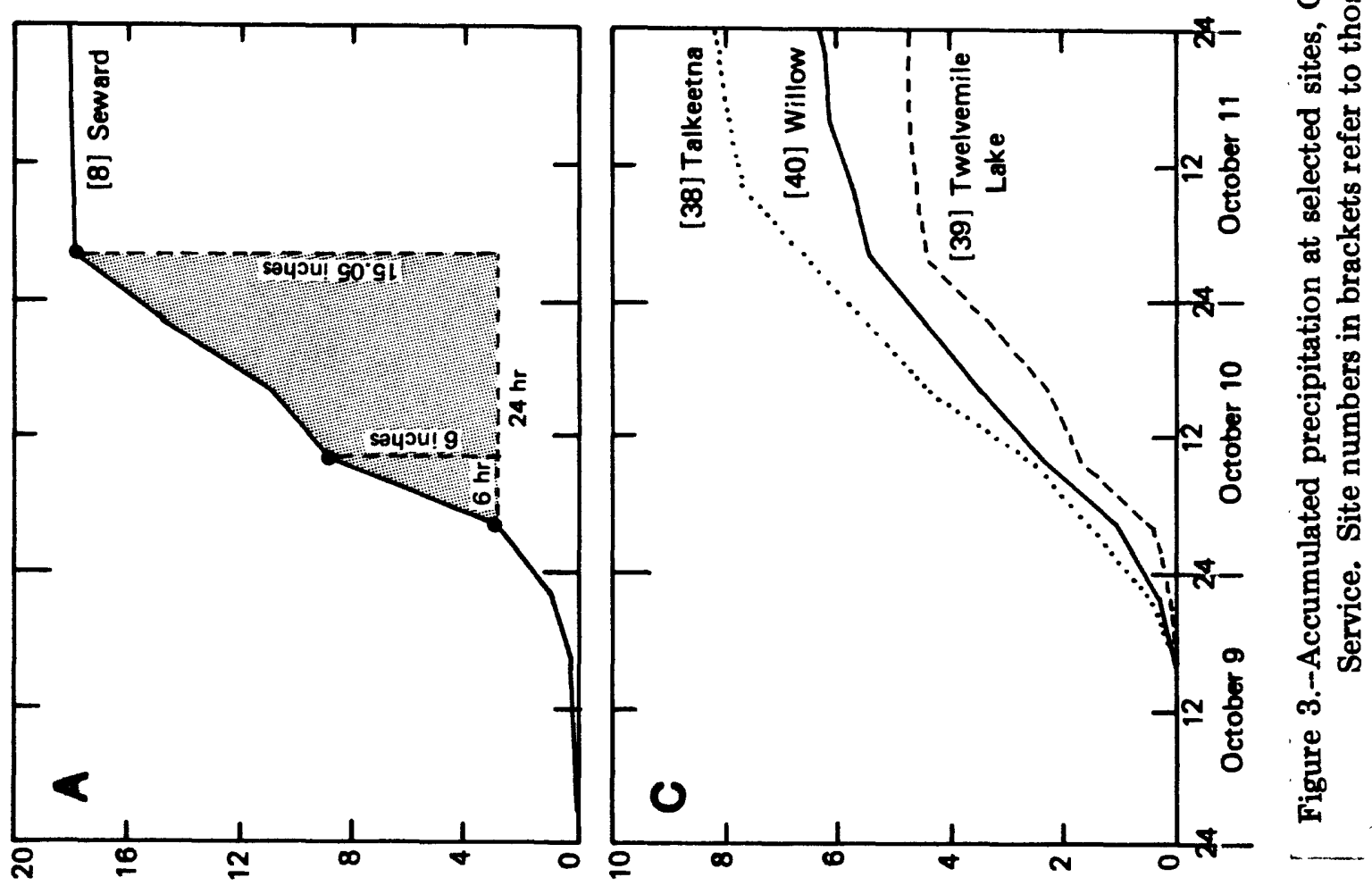

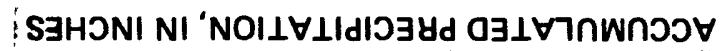


1963 and Nibler, 1986). The precipitation at three sites in the area is graphed in figure $3 \mathrm{C}$.

The freezing levels during this storm period were at comparatively high altitudes. For example, freezing levels during the early morning of October 10 were at about 7,000 ft at Fairbanks and Anchorage. In contrast, freezing levels the night of October 8 were at ground level at Fairbanks and at 3,000 ft over Anchorage. Normally, most precipitation in October at higher altitudes occurs as snow. (See Nibler, 1986.)

\section{DISCHARGE DATA}

\section{Peak Stage and Discharge Table}

Peak stages and discharges were determined at many gaging stations, creststage gage sites, and miscellaneous sites affected by the October floods. Table 2 lists the data for these sites. The site numbers shown in table 2 correspond to the numbers on figures $1,4,6,8$, and 10 , which show site locations. Table 2 includes information about: (1) the downstream order station number; (2) the stream name and location; (3) drainage area; (4) the period of record during which systematic collection of discharge data occurred, and at a few sites, the year that peak discharge data were collected during prior outstanding floods; (5) date, peak stage (gage height), and discharge for the maximum flood previously documented; and (6) the date, peak stage (gage height), and discharge for the October 1986 flood.

The indicated recurrence interval, or frequency, of the floods (table 2) is the average number of years between floods with maximum discharges equal to or greater than those of October 10-12, 1986. A given recurrence interval does not imply that it will be that many years before a flood of that magnitude occurs again. In fact, floods of similar or greater magnitude can occur in the same year or the following year. Recurrence intervals are the reciprocal of the probability of a flood event, of a given size or larger, occurring during any given year. That is, a 100 -year flood has a 0.01 probability or 1 percent chance of occurring in any one year. All frequencies, or recurrence intervals, were determined from station data using log-Pearson III frequency analysis procedures described by the Water Resources Council (1981). Individual station frequency curves used the "skew" and "standard deviation" statistics computed by Lamke (1979). Most of the stations have short periods of record; the recurrence interval for these stations is shown as a range in years or qualified as "about"; a ratio of the October maximum to the computed 100-year flood discharge is shown for three stations, Montana (57) and Caswell Creeks (60) and Deshka River (65), with recurrence intervals greater than 100 years. (Hereafter, the site numbers, for example (57) and (60), are included for those sites mentioned in the description of the flood. Site numbers for the precipitation measurement sites listed in table 1 are bracketed; for example, Homer Airport [10].)

\section{Discharge Data for October 1986}

Discharge data for October 1986 were computed for six selected streamgaging stations, at which the peak of the period of record occurred during the month. Dally discharges for the month along with gage height and discharge data sufficient to prepare hydrographs for the flood period are presented later in 
Table 2.--Summary of flood stages and discharges for the flood of October 10-12, 1986

ISite numbers refer to those on figures $1,4,6,8$, and 10 ;

mil 2 , quare miles: $w$. y.. water year; $\mathrm{ft}^{3} / \mathrm{s}$, cubic feet per second]

\begin{tabular}{|c|c|c|c|c|c|c|c|c|c|c|c|}
\hline \multirow[b]{2}{*}{$\begin{array}{l}\text { Site. } \\
\text { No. }\end{array}$} & \multirow[b]{2}{*}{$\begin{array}{l}\text { Permanent } \\
\text { etation } \\
\text { number }\end{array}$} & \multirow[b]{2}{*}{$\begin{array}{l}\text { Stream name and } \\
\text { location }\end{array}$} & \multirow[b]{2}{*}{$\begin{array}{l}\text { Drainage } \\
\text { ares } \\
\left(m 1^{2}\right)\end{array}$} & \multirow[b]{2}{*}{$\begin{array}{c}\text { Annual } \\
\text { peak flow } \\
\text { records } \\
\text { (w.y.) }\end{array}$} & \multicolumn{3}{|c|}{$\begin{array}{l}\text { Maximum discharge } \\
\text { proviousiy known }\end{array}$} & \multicolumn{4}{|c|}{$\begin{array}{l}\text { Maximum discharge } \\
\text { in flood period }\end{array}$} \\
\hline & & & & & Date & $\begin{array}{c}\text { Gage } \\
\text { helght } \\
\text { (foet) }\end{array}$ & $\begin{array}{l}\text { Dis- } \\
\text { charge } \\
\left(\mathrm{ft}^{3} / \mathrm{s}\right)\end{array}$ & Day & $\begin{array}{c}\text { Gage } \\
\text { height } \\
(\text { Eeet) }\end{array}$ & $\begin{array}{c}\text { Dis- } \\
\text { charge } \\
\left(\mathrm{ft}^{\prime} / \mathrm{s}\right)\end{array}$ & $\begin{array}{l}\text { Recur- } \\
\text { rence } \\
\text { interval } \\
\text { (years) }\end{array}$ \\
\hline 1 & 15216000 & $\begin{array}{l}\text { Power Creok noar } \\
\text { Cordova }\end{array}$ & 20.5 & $1948-$ & $8-25-49$ & 7.65 & 5,540 & 21 & 5.89 & 3,040 & 3 \\
\hline 2 & 15225998 & $\begin{array}{l}\text { Solomon Gulch Bypas: } \\
\text { near Valdez }\end{array}$ & (a) & 1986- & $9-20-86$ & 8.25 & 1,290 & 11 & 12.11 & 3.370 &.- \\
\hline 3 & 15226000 & $\begin{array}{l}\text { Solomon Gulch near } \\
\text { Valdez }\end{array}$ & 19.7 & $1950-56$, & $8-4-51$ & b6. 50 & 2,420 & 11 & $\cdots$ & $c 2,230$ & -- \\
\hline 4 & 15236200 & $\begin{array}{l}\text { Shakespeare Creok at } \\
\text { Whittior }\end{array}$ & d1.61 & $\begin{array}{c}1970-80, \\
84-\end{array}$ & $9-13-7 \theta$ & 12.61 & 620 & 11 & 11.2 & 475 & 3 \\
\hline 5 & 15237360 & $\begin{array}{l}\text { San Juan River noar } \\
\text { Soward }\end{array}$ & 12.4 & $1986-$ & $9-8-86$ & 7.88 & 1,730 & 11 & 6.80 & 1.140 & -- \\
\hline 6 & $\cdots$ & $\begin{array}{l}\text { Godwin Creok noar } \\
\text { Soward }\end{array}$ & 13.8 & $\cdots$ & $\cdots$ & --- & $\cdots$ & 11 & 44.108 & $8 \cdot 30.000$ & -- \\
\hline 7 & $-\cdot$ & $\begin{array}{l}\text { Sawmill Creok noar } \\
\text { Sorfard }\end{array}$ & 7.85 & $\cdots$ & --- & --- & -- & 11 & 51.30 & 2,900 & $\cdots$ \\
\hline 8 & $-\cdot$ & $\begin{array}{l}\text { Sawmill Creok at Nash } \\
\text { Road noar Soward }\end{array}$ & 10.6 & -- & -- & -- & --- & 11 & 129.99 & 884,000 & -- \\
\hline 8 & $\cdots$ & $\begin{array}{l}\text { Resurrection River at } \\
\text { Exit Glacier bridge }\end{array}$ & 106 & $-\infty$ & 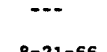 & -- & 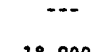 & 11 & $\$ 353.28$ & (h) & -- \\
\hline 10 & 15237700 & $\begin{array}{l}\text { Resurrection River at } \\
\text { Seward }\end{array}$ & 169 & 1965-67 & $8-21-66$ & b10.68 & 18,900 & 11 & 131.02 & 819,000 & -- \\
\hline 21 & 15238000 & Lost Creok near Seward & $d 8.42$ & $\begin{array}{l}1949, \\
63-72,76\end{array}$ & $6^{9-20-76}$ & 12.30 & 820 & 11 & (h) & 8.14 .000 & $\cdots$ \\
\hline 12 & $\cdots$ & $\begin{array}{l}\text { Grouse Creok near } \\
\text { Soward } \\
\text { Lost Craok at Bridge }\end{array}$ & $\begin{array}{r}4.78 \\
23.6\end{array}$ & $\cdots$ & $\cdots$ & $\cdots$ & $\cdots$ & 11 & $\begin{array}{r}84.65 \\
\$ 143.00\end{array}$ & $\begin{array}{r}1,890 \\
-8,500\end{array}$ & $\cdots$ \\
\hline 14 & $\cdots$ & $\begin{array}{l}600 \text { naar Soward } \\
\text { Salmon Creok noar }\end{array}$ & 7.10 & $\cdots$ & -- & $\cdots$ & 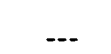 & 21 & 50.03 & 4.200 & -- \\
\hline 15 & $\cdots$ & $\begin{array}{l}\text { Seward } \\
\text { Cloar Crook noar }\end{array}$ & --- & -- & -- & $\cdots$ & $\cdots$ & 11 & $\$ 43.01$ & 52,800 & $\cdots$ \\
\hline 16 & $\cdots$ & $\begin{array}{l}\text { Seward } \\
\text { Salmon Crook at Nash } \\
\text { Road noar Seward }\end{array}$ & $\cdots$ & --- & -- & --- & $\cdots$ & 11 & $\$ 23.44$ & $8 J 10,300$ & $\cdots$ \\
\hline 17 & -- & $\begin{array}{l}\text { Rudolph Creek near } \\
\text { Seward }\end{array}$ & 1.00 & -- & -- & -- & -- & 11 & -- & 1.020 & $\cdots$ \\
\hline 18 & 15238500 & Lowell Creak at Seward & 4.02 & $1966-68$ & $8-21-66$ & (k) & 1,200 & 11 & (k) & (h) & $\cdots$ \\
\hline-- & -- & $\begin{array}{l}\text { Spruce Creok above debris } \\
\text { aval anche near Seward }\end{array}$ & 8.98 & $\cdots$ & -- & $\cdots$ & $\cdots$ & 11 & -- & 5,420 &.- \\
\hline 19 & 25238600 & $\begin{array}{l}\text { Spruce Croek naar } \\
\text { Seward }\end{array}$ & 9.26 & 1966- & $9-25-82$ & 9.46 & 3,420 & 11 & $\mathrm{~m} 13.96$ & $\mathrm{~m} 13,600$ & -- \\
\hline 20 & 15238648 & $\begin{array}{l}\text { Uppor Nuka River near } \\
\text { Homer }\end{array}$ & ne3 & 1985- & $9-27-85$ & 4.42 & 709 & 11 & 4.48 & 777 & -- \\
\hline 21 & 25238820 & $\begin{array}{l}\text { Barbara Crook near } \\
\text { Seldovia }\end{array}$ & 20.7 & $1973-$ & $11-29-83$ & 6.08 & 2,050 & 10 & 4.26 & 1,5901 & $10-15$ \\
\hline 22 & 15238990 & $\begin{array}{l}\text { Upper Bradley Rivar } \\
\text { near Homer }\end{array}$ & n10 & 1980- & $8-27-86$ & 9.30 & 2.150 & 10 & 9.86 & 2,530 & $5-10$ \\
\hline 23 & 15239000 & $\begin{array}{l}\text { Bradley River near } \\
\text { Homer }\end{array}$ & n54 & 1958- & $8-10-79$ & 9.46 & 6,020 & 10 & 10.80 & 8,800 & 50 \\
\hline 24 & 15239050 & $\begin{array}{l}\text { Bradley River } \\
\text { tributary near Bomer }\end{array}$ & 9.25 & $1980-$ & $7-11-80$ & 8.05 & 765 & 10 & 8.53 & 1,120 & $10-15$ \\
\hline 25 & 15239070 & $\begin{array}{l}\text { Brodloy River noar } \\
\text { tidewater noar Homer }\end{array}$ & n82 & $1984-$ & $8-28-86$ & 10.32 & 4,160 & 11 & 13.73 & 11,000 & $\cdots$ \\
\hline 26 & 15239500 & Fritz Croek near Homer & 10.4 & 1963- & $10-22-80$ & b18.53 & 852 & p15 & p10.85 & p112 & $<2$ \\
\hline 27 & 15239900 & $\begin{array}{l}\text { Anchor River near } \\
\text { Anchor Point }\end{array}$ & 137 & $\begin{array}{l}1966-74,1 \\
79-\end{array}$ & $11-28-83$ & 7.42 & 6,050 & 10 & 5.17 & 2,210 & 5 \\
\hline 28 & 15240000 & $\begin{array}{l}\text { Anchor River at Anchor } \\
\text { Point }\end{array}$ & 224 & $1954-66,1$ & $11-30-83$ & 8.51 & 11,000 & 10 & 6.30 & 5,900 & 25 \\
\hline 28 & 15243900 & Snow River near Seward & 128 & $\begin{array}{l}1967,70 \\
74,77,86\end{array}$ & $8-31-67$ & b42.6 & $q 55,000$ & 11 & 1480.24 & 17,500 & $\cdots$ \\
\hline 30 & $\cdots$ & $\begin{array}{l}\text { Sixtoon Mile Craek } \\
\text { near Soward }\end{array}$ & 3.19 & $\cdots$ & $\cdots$ & $\cdots$ & $\ldots$ & 11 & 21.44 & 2,550 & $\cdots$ \\
\hline 31 & $\cdots$ & $\begin{array}{l}\text { Snow River at Seward } \\
\text { Hi ghway noar Soward }\end{array}$ & 165 & $\begin{array}{l}1970,74 \\
77,85,86\end{array}$ & $9-20-74$ & $=-$ & $q 28,300$ & 11 & $\cdots$ & 815,400 & -- \\
\hline 32 & 15243950 & $\begin{array}{l}\text { Porcuptno Croak near } \\
\text { Primrose }\end{array}$ & 16.8 & 1963- & 0-18-82 & 213.44 & 3,350 & 11 & 13.03 & 4,000 & 80 \\
\hline 33 & 15248000 & $\begin{array}{l}\text { Trail River noar } \\
\text { Lawing }\end{array}$ & 181 & $1948-77$ & $9-18-67$ & 11.93 & 7.480 & 12 & 9.7 & 84,800 & 3 \\
\hline 34 & 15251800 & $\begin{array}{l}\text { Quartz Creok at } \\
\text { Gilpatrick: }\end{array}$ & 9.41 & $\begin{array}{l}1963-70 \\
1976\end{array}$ & $10-6-69$ & 11.54 & 633 & 11 & (h) & 897 & 50 \\
\hline 35 & 15254000 & $\begin{array}{l}\text { Croecent Creok noar } \\
\text { Cooper Landing }\end{array}$ & 31.7 & $1950-83$ & $10-9-69$ & 12.73 & 1,500 & 11 & 11.73 & 680 & 10 \\
\hline 36 & -- & $\begin{array}{l}\text { Quartz Creok near } \\
\text { Cooper Landing }\end{array}$ & 111 & -- & -- & -- & -- & 11 & -- & 2,400 & -- \\
\hline 37 & 15258000 & $\begin{array}{l}\text { Kenal River at Cooper } \\
\text { Landing }\end{array}$ & 634 & $1848-$ & $9-21-74$ & 17.18 & $q 23,100$ & 14 & 13.84 & 13.400 & 4 \\
\hline 38 & 15266300 & $\begin{array}{l}\text { Konai River at } \\
\text { Soldotna }\end{array}$ & 2,010 & 1965- & $9-9-77$ & 13.45 & 33,700 & 18 & 10.26 & 16,300 & $<2$ \\
\hline 39 & 15266500 & $\begin{array}{l}\text { Beaver Creek near } \\
\text { Konal }\end{array}$ & 51 & $\begin{array}{l}1968-78 \\
80-83.85\end{array}$ & $5-8-72$ & b10.55 & 598 & 11 & 9.43 & 700 & 20 \\
\hline 40 & 15271000 & $\begin{array}{l}\text { Sixm1 le Creok noar } \\
\text { Hope }\end{array}$ & 234 & $\begin{array}{l}1970 \\
1980-\end{array}$ & $7-12-80$ & 13.22 & 8.070 & 10 & 12.73 & 6.320 & 5 \\
\hline
\end{tabular}


Table 2.--Summary of flood stages and discharge for the flood of October 10-12, 1986--Continued

\begin{tabular}{|c|c|c|c|c|c|c|c|c|c|c|c|}
\hline \multirow[b]{2}{*}{$\begin{array}{l}\text { Size } \\
\text { No. }\end{array}$} & \multirow[b]{2}{*}{$\begin{array}{l}\text { Pormanent } \\
\text { seation } \\
\text { number }\end{array}$} & \multirow[b]{2}{*}{$\begin{array}{l}\text { Stroam neme and } \\
\text { locetion }\end{array}$} & \multirow[b]{2}{*}{$\begin{array}{l}\text { Dratnese } \\
\left.\cos ^{2}\right) \\
\left.(\operatorname{lil})^{2}\right)\end{array}$} & \multirow[b]{2}{*}{$\begin{array}{l}\text { Annuel } \\
\text { peat Ilow } \\
\text { records } \\
\text { (w. y.) }\end{array}$} & \multicolumn{3}{|c|}{$\begin{array}{l}\text { Maximum discheres } \\
\text { provl ous ly known }\end{array}$} & \multicolumn{4}{|c|}{$\begin{array}{l}\text { Maximupd dischere: } \\
\text { in Elood porlod }\end{array}$} \\
\hline & & & & & Date & $\begin{array}{l}\text { Gece } \\
\text { hotche } \\
\text { (foee) }\end{array}$ & $\begin{array}{l}\text { Dis:- } \\
\operatorname{char}^{\circ}\left(2 e^{\circ} / 8\right)\end{array}$ & Day & $\begin{array}{l}\text { Gase } \\
\text { holcht } \\
\text { (foot) }\end{array}$ & $\begin{array}{c}\text { D1:- } \\
\operatorname{charg} g^{\circ}\end{array}$ & $\begin{array}{l}\text { Recur- } \\
\text { rence } \\
\text { Ineorvel } \\
\text { (yours) }\end{array}$ \\
\hline 41 & 25272530 & $\begin{array}{l}\text { Callfornita Croek at } \\
\text { Girdwood }\end{array}$ & 6.96 & $1867-$ & $10-6-69$ & 20.20 & 600 & 12 & 15.81 & 250 & 3 \\
\hline 42 & 15274600 & $\begin{array}{l}\text { Campboll Crook noar } \\
\text { Sponard }\end{array}$ & 69.7 & $1866-$ & $8-23-81$ & b3.54 & 451 & 12 & 20.89 & 472 & 7 \\
\hline 43 & 15276000 & $\begin{array}{l}\text { Ship Crook noar } \\
\text { Anchorage }\end{array}$ & 60.5 & $1047-$ & $6-21-49$ & b3.44 & 1.860 & 12 & 6.08 & 1,310 & 15 \\
\hline 44 & 25277600 & $\begin{array}{l}\text { Eest Fork Eklutna } \\
\text { Crook noar Palmer }\end{array}$ & 38.2 & $\begin{array}{c}1861-62 \\
65-\end{array}$ & $9-12-61$ & b3.86 & 1,320 & 21 & 9.19 & 1,500 & e6 \\
\hline 45 & 25277800 & $\begin{array}{l}\text { Wost Fork Eklutna } \\
\text { Croek noar Polmer }\end{array}$ & 25.4 & $\begin{array}{c}1961-62 \\
05-\end{array}$ & $6-28-62$ & b3.8a & 1.670 & 12 & 9.05 & 606 & $<2$ \\
\hline 48 & 15283500 & Esk a Creak near Sucton & 13.4 & 1966 & $6-20-71$ & b26.82 & 2,660 & 11 & 6.74 & 223 & 3 \\
\hline 47 & 25285000 & $\begin{array}{l}\text { Wes1lle Croek noer } \\
\text { Palmar }\end{array}$ & 26.8 & 1971 & $8-20-71$ & 17.74 & 700 & 11 & 9.56 & 89 & $<2$ \\
\hline 46 & 15285200 & $\begin{array}{l}\text { Wasilla Croek noar } \\
\text { Wesille }\end{array}$ & 39.5 & 1860- & 8-16-80 & 3.46 & 243 & 11 & 2.47 & 92 & $<2$ \\
\hline 49 & 15290000 & $\begin{array}{l}\text { Lietle Susitne River } \\
\text { noar Palmer }\end{array}$ & 62.8 & $1948-$ & $6-10-71$ & $e 13$ & 7.640 & 11 & 8.17 & 2.510 & 3 \\
\hline so & 15280100 & $\begin{array}{l}\text { Litele Susitna RIver } \\
\text { near Bouston }\end{array}$ & 166 & $\begin{array}{l}1980-81, \\
1984-\end{array}$ & $9-16-60$ & 15.31 & 3.200 & 12 & 15.30 & 3,600 & $10-25$ \\
\hline $\begin{array}{l}51 \\
52\end{array}$ & $\begin{array}{l}15290200 \\
15292000\end{array}$ & $\begin{array}{l}\text { Nancy Lake tribueary } \\
\text { near Willow } \\
\text { Susitne River at Gold }\end{array}$ & $\begin{array}{l}6.00 \\
6,160\end{array}$ & $\begin{array}{l}2980, \\
2983:- \\
2950-\end{array}$ & $\begin{array}{r}6-21-80 \\
6-7-64\end{array}$ & $\begin{array}{l}12.42 \\
26.58\end{array}$ & 295 & 11 & 23.21 & 36,500 & $\begin{array}{l}10-25 \\
<2\end{array}$ \\
\hline 53 & 25292400 & $\begin{array}{l}\text { Creek } \\
\text { Chulitne RIver noar }\end{array}$ & 2,570 & $1958-77$ & $7-20-67$ & 22.48 & 75,900 & 11 & 11.02 & 36,500 & $10-15$ \\
\hline 54 & 15292700 & $\begin{array}{l}\text { Tolkeolna } \\
\text { Ialkeotne River noar } \\
\text { Iolkeotne }\end{array}$ & 2,006 & $\begin{array}{l}1979- \\
1964-\end{array}$ & $6-20-72$ & 26.35 & 67,400 & 11 & 17.38 & 75,700 & 40 \\
\hline 55 & 15292780 & $\begin{array}{l}\text { Susiena River et } \\
\text { Sunshine }\end{array}$ & 11,200 & 1971:- & $8-20-71$ & b62.10 & 200,000 & 11 & 16.5 & 185,000 & $25-50$ \\
\hline 56 & $\cdots$ & $\begin{array}{l}\text { Rabideux Creek noar } \\
\text { Sunshine }\end{array}$ & 27 & -- & -- & -- & -- & 11 & 19.23 & 2.700 & $-\infty$ \\
\hline 57 & 15292800 & $\begin{array}{l}\text { Montana Craek near } \\
\text { Hontana }\end{array}$ & 164 & $1963-72$ & $8-10-71$ & 12.96 & 6,970 & 11 & $\$ 20.0$ & 15,300 & $t 1.3$ \\
\hline 58 & 15292800 & $\begin{array}{l}\text { Goose Craek near } \\
\text { Montane }\end{array}$ & MOTE & $\begin{array}{l}1963-71, \\
1984-\end{array}$ & $0-10-72$ & b19.68 & 3,270 & 21 & 5.80 & 7,000 & $\cdots$ \\
\hline 59 & 25292990 & $\begin{array}{l}\text { Shoop Creak near } \\
\text { willow }\end{array}$ & NOTE & 1884 & $8-25-84$ & 2.82 & 1.810 & 12 & 5.39 & 6,200 & -- \\
\hline 60 & 25293000 & $\begin{array}{l}\text { Caswell Croek noar } \\
\text { Caswell }\end{array}$ & 29.6 & 2963- & $8--85$ & 12.89 & 207 & 11 & 18.00 & 860 & 21.43 \\
\hline 81 & 15293700 & $\begin{array}{l}\text { Litele Willow Craek } \\
\text { neer Kashwithe }\end{array}$ & 155 & $1080-$ & $8-12-65$ & 23.30 & 2,500 & 21 & 15.25 & 6,500 & e30 \\
\hline 62 & 25294005 & $\begin{array}{l}\text { Willow Creok near } \\
\text { Willow }\end{array}$ & 166 & 1979- & $7-28-80$ & b8.80 & 4.450 & 11 & 9.01 & 12,000 & eso \\
\hline 83 & 15294010 & $\begin{array}{l}\text { Decoption Croat neer } \\
\text { Willow }\end{array}$ & 48 & $1978-85$ & $6-21-80$ & 8.44 & 751 & 11 & 8.89 & 800 & $10-15$ \\
\hline 64 & 15294025 & $\begin{array}{l}\text { Moose Croeak naar } \\
\text { Talkeotne }\end{array}$ & 52.3 & 1972- & $7-10-61$ & b26.73 & 2,500 & 11 & 31.80 & 5,790 & 100 \\
\hline 65 & 15294100 & $\begin{array}{l}\text { Deshike River near } \\
\text { Willow }\end{array}$ & 592 & 1979- & $11-13-79$ & 6.26 & 9,920 & 12 & 23.5 & 48,000 & $t 1.3$ \\
\hline 66 & 25294300 & $\begin{array}{l}\text { Skwentna RLVer near } \\
\text { Skwantine }\end{array}$ & .250 & $1960-82$ & $6-09-77$ & 15.09 & 51.600 & 11 & 17.3 & 69,000 & 100 \\
\hline 87 & 15294345 & $\begin{array}{l}\text { Yontene River noar } \\
\text { Susitne Station }\end{array}$ & 6.160 & 1981- & $8-13-81$ & 18.61 & 118,000 & 12 & 18.21 & 130.000 & 10 \\
\hline 68 & 15294350 & $\begin{array}{l}\text { Susiena River at } \\
\text { Susiene Station }\end{array}$ & 19.400 & 1975- & $\begin{array}{l}7-29-60 \\
8-16-81\end{array}$ & $\begin{array}{l}20.26 \\
20.27\end{array}$ & $\begin{array}{l}230,000 \\
230,000\end{array}$ & 12 & 22.58 & 312,000 & 40 \\
\hline 69 & 15294410 & $\begin{array}{l}\text { Capps Craek bolow North } \\
\text { Capps Croak noer } \\
\text { Tyonok }\end{array}$ & 10.5 & $1980-85$ & $8-15-82$ & 7.60 & 550 & 10 & 11.30 & $>1,200$ & (u) \\
\hline 70 & 15294450 & $\begin{array}{l}\text { Chuitne River noar } \\
\text { Tyonek }\end{array}$ & 131 & 1976- & $9-25-82$ & 10.07 & 4.800 & 10 & 16.46 & $>10,000$ & (u) \\
\hline
\end{tabular}

(NOTE: Flood flows from Shoop and Goose Croeks intermingle; combined drainage orea is 157 al ${ }^{2}$.)

About.

Greeter than value show.

Less then value show.

Resulated.

At different datum or localion.

Maximum datly discherse.

Revised from proviously publishod annual reports.

Discherso alfoctod by landslido, dobrls rlow.

totel discherse:

Toter discherc:

Includes water diverted from Box Canyon Creok.

Includes water diverted from Box Canyostean diversion dam in 1966 and $0.7 \mathrm{fe}$ below in 1986.

Stese and discherse effected by debris-avalenche release flood.

Dretnese

Peat occurred during period of questionable sace-holght record, Oct. 10-15. Gage-height shown was the

hishest recorded in the period, but - hisher poak dischargo may have occurred earlier.

Glecter-darmed lake outburst flood.

Backwater from Kenal Lake.

Maximum stage might have occurred prior to maximum disharge.

Ratio to estimated discharse of a flood with a 100-year recurrence interval.

Probably greater than 100-year flood discharge; extraordinary channel changes. 
tables 3, 4, and 5; discharge hydrographs for October 9-15, were graphed and also are shown later in the report. Alaska Daylight Savings Time (ADST) is used throughout the report.

\section{FLOOD DESCRIPTION}

The greatest rainfall intensity in the lower Kenai Peninsula occurred the afternoon and night of October 9 (see Homer [10], fig. 5); the storm then moved eastward to Seward, where the highest intensities were at mid-morning of october 10 (fig. 3A). The center of heavy rainfall then moved northward to the Anchorage area on the afternoon and evening of October 10 (see fig. 9). In the Susitna River basin, still farther north, the high intensity precipitation began the morning of october 10 and continued throughout the day (fig. 3C). To the southeast near Cordova, the highest 6 -hour intensities occurred during midmorning of October 11, 1986 (fig. 3D). The description of the flood will generally follow the above sequence.

\section{Kenai Peninsula}

\section{Bradley Lake and Homer Area Streams}

The most extreme flooding documented in the lower Kenai Peninsula was in the Bradley River basin (fig. 4). Stream stage and discharge at Upper Nuka River (20), Upper Bradley River (22), Bradley River (23), Bradley River tributary (24), and Bradley River near tidewater (25) exceeded all prior maximums. These stations have seven or fewer years of flood record except for Bradley River (23), which has 27 years. Upper Bradley River (22), which originates at Nuka Glacier, had a peak discharge of $2,530 \mathrm{ft} / \mathrm{s}$ with an estimated recurrence interval of about 10 years. The peak recurrence interval downstream at Bradley River (23), at the downstream end of Bradley Lake, was 50 years. Much of the flood flow probably was from Kachemak Creek, which begins at Kachemak Glacier; this stream has about 80 percent of the drainage area tributary to Bradley Lake.

Discharge data for Upper Bradley River (22) are presented in table 3 and a discharge hydrograph is shown in figure 5. Precipitation at Homer [10] was less than in the Bradley River drainage (fig. 2), but the timing of the highest intensities probably coincides. The peak discharge occurred just after midnight October 9. The increase in discharge ( $f i g .5$ ) on the afternoon of October 10 might have been caused by fringes of the storm around Seward [8] (figs. 3 and 5) or the rise might be due to snow and glacier melt resulting from above-freezing temperatures at high altitudes. The gaging station is at an altitude of about $1,200 \mathrm{ft}$ above sea level.

Southwest of Bradley Lake, about 14.3 in. of rain fell at Tutka Bay Lagoon [9] during the 3-day storm period; this was the second highest recorded rainfall in southcentral Alaska. The $6 \mathrm{p} . \mathrm{m}$. reading on 0ctober 10 was 10.31 in.; the gage is read once daily at $6 \mathrm{p.m}$. (ADST). (See U.S. Dept. of Commerce, 1986.) However, peak discharge at Barbara Creek near Seldovia (21), which is about $9 \mathrm{mi}$ northwest of the Tutka Bay precipitation gage, was not as high as that of November 29, 1983. The recurrence interval of the peak discharge, $1,590 \mathrm{ft}^{3} / \mathrm{s}$, was about 10 to 15 years. West of Homer, along the Sterling Highway, peak discharges at gaging station, Anchor River near Anchor Point (27) and Anchor 


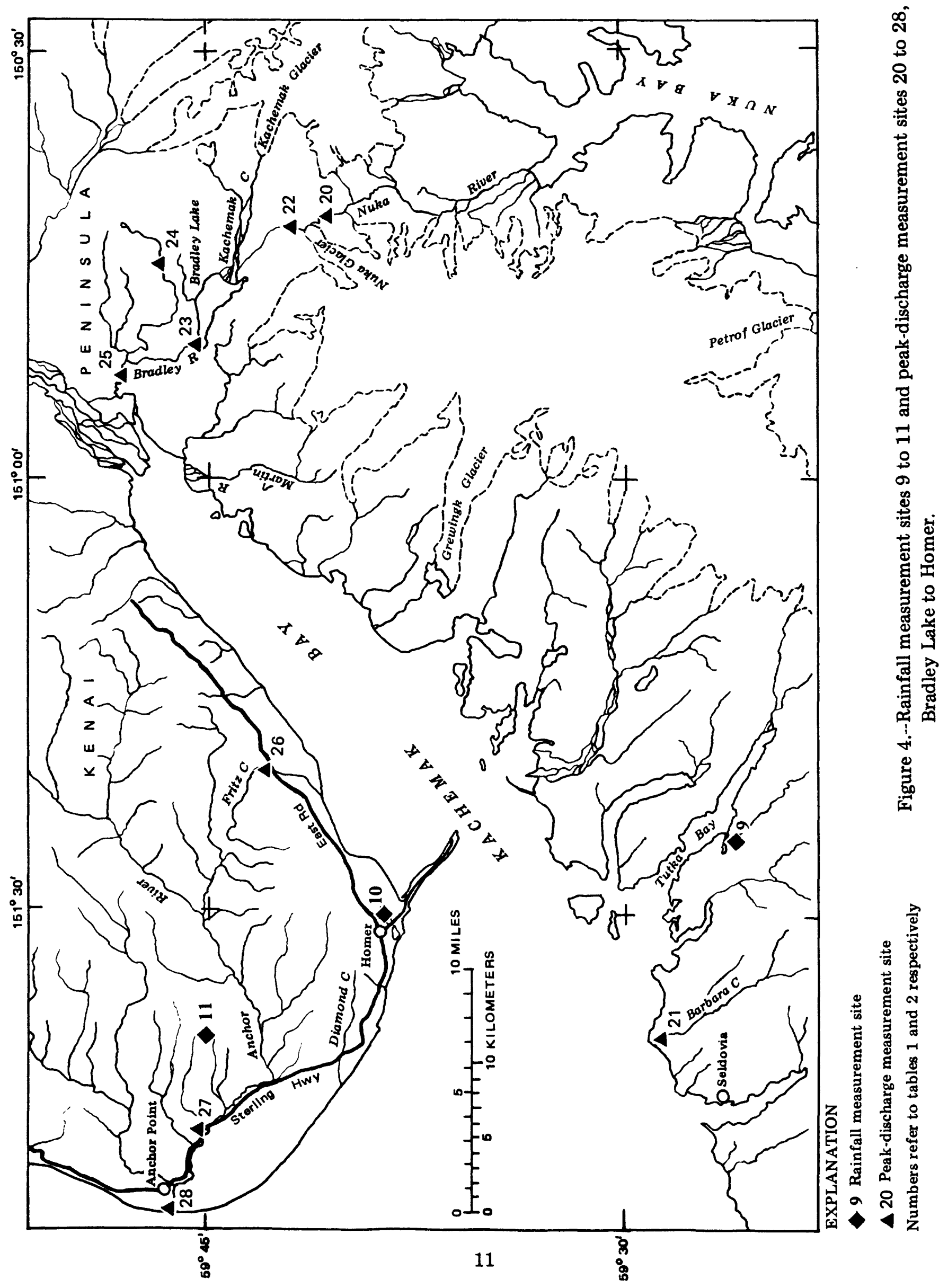


Table 3.--Discharge data for Upper Bradley River during October 1986

(22)

15238990 UPPER BRADLEY RIVER NEAR BOMER

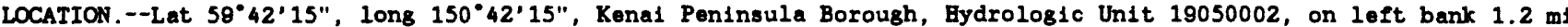
downstream of Nuka Giacier terminus, $2.5 \mathrm{ml}$ upstream of mouth at Kachemak Creek, $3.5 \mathrm{ml}$ southeast of Bradley Lake, and $29 \mathrm{ml}$ east of Homer.

DRAINAGE AREA.--About $10 \mathrm{mi}^{2}$. Drainage area varies according to position of terminus of Nuka Glacier; $1 \mathrm{t}$ became amaller on June 1983 as snowmelt runoff from Nuka Glacier began to flow again into both the Nuka and Bradley River dralnage basins.

GAGE-HEIGHT RECORD. --Water-stage recorder graph except Oct. 1-6. Elevation of gage 1s 1,200 ft above National Geodetic Vertical Datum of 1929, from topographic map.

DISCAARGE RECORD.--Stage-djscharge relation defined by current-meter measurements below $450 \mathrm{ft}^{3} / \mathrm{s}$ and $\mathrm{slope-area}$ measurement at $2,530 \mathrm{ft}^{3} / \mathrm{s}$. Discharge Oct. 1-6 estimated by comparison with nearby station records.

MAXIMUM FOR PRIOR FLOOD RECORD.--(Water years 1980-86), 2,150 ft $3 / \mathrm{s}$, Aug. 27, 1986, 8age he1ght, $9.30 \mathrm{ft}$; maximum gage he1ght, $13.09 \mathrm{ft}$, Dec. 17, 1981, backwater from $1 \mathrm{ce}$.

MAXIMUM FOR OCTOBER FLOOD.-- 2,530 ft $3 / \mathrm{s}, 0030$ hours Oct, 10, gage he1ght, $9.86 \mathrm{ft}$, from recorder, $9.8 \mathrm{ft}$, from high-water mark.

Mean discharge, In cublc feet per second, October 1986

\begin{tabular}{|c|c|c|c|c|c|c|c|c|c|c|c|}
\hline Day & $\begin{array}{c}\text { D1s- } \\
\text { charge } \\
\end{array}$ & Day & $\begin{array}{c}\text { D1s- } \\
\text { charge }\end{array}$ & Day & $\begin{array}{c}\text { D1s- } \\
\text { charge }\end{array}$ & Day & $\begin{array}{c}\text { D1s- } \\
\text { charge } \\
\end{array}$ & Day & $\begin{array}{c}\text { D1s- } \\
\text { charge }\end{array}$ & Day & $\begin{array}{c}\text { D1s- } \\
\text { charge }\end{array}$ \\
\hline $\begin{array}{l}1 \\
2 \\
3 \\
4 \\
5\end{array}$ & $\begin{array}{l}170 \\
140 \\
400 \\
280 \\
190\end{array}$ & $\begin{array}{r}6 \\
7 \\
8 \\
9 \\
10\end{array}$ & $\begin{array}{r}130 \\
94 \\
60 \\
521 \\
1,440\end{array}$ & $\begin{array}{l}11 \\
12 \\
13 \\
14 \\
15\end{array}$ & $\begin{array}{r}1,070 \\
730 \\
614 \\
186 \\
127\end{array}$ & $\begin{array}{l}16 \\
17 \\
18 \\
19 \\
20\end{array}$ & $\begin{array}{l}81 \\
55 \\
47 \\
43 \\
42\end{array}$ & $\begin{array}{l}21 \\
22 \\
23 \\
24 \\
25\end{array}$ & $\begin{array}{r}101 \\
83 \\
48 \\
40 \\
36\end{array}$ & $\begin{array}{l}26 \\
27 \\
28 \\
29 \\
30 \\
31\end{array}$ & $\begin{array}{l}32 \\
29 \\
27 \\
24 \\
22 \\
22\end{array}$ \\
\hline
\end{tabular}

Gage helght, In feet: discharge, in cublc feet per second; at indicated date and time in 1986

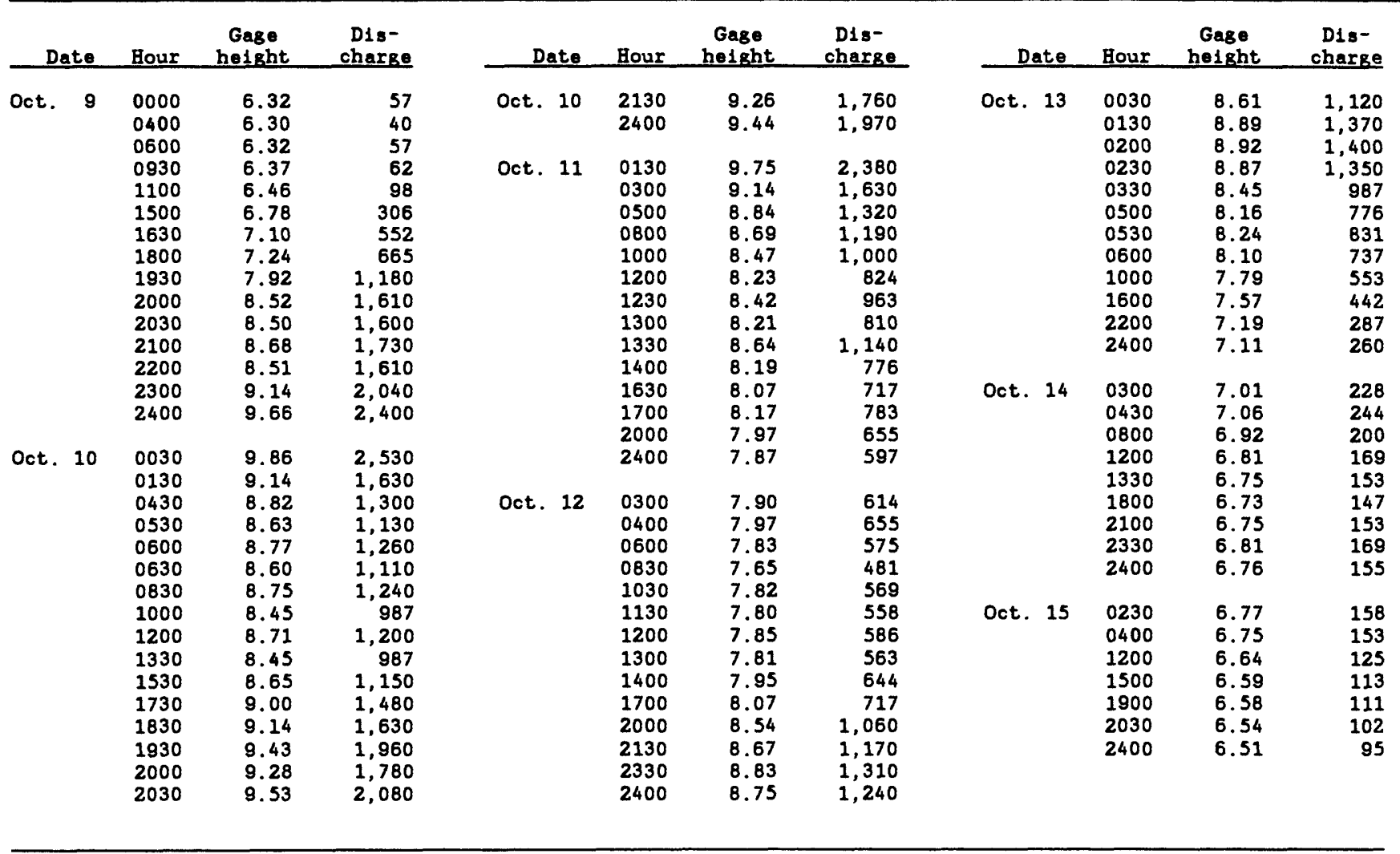




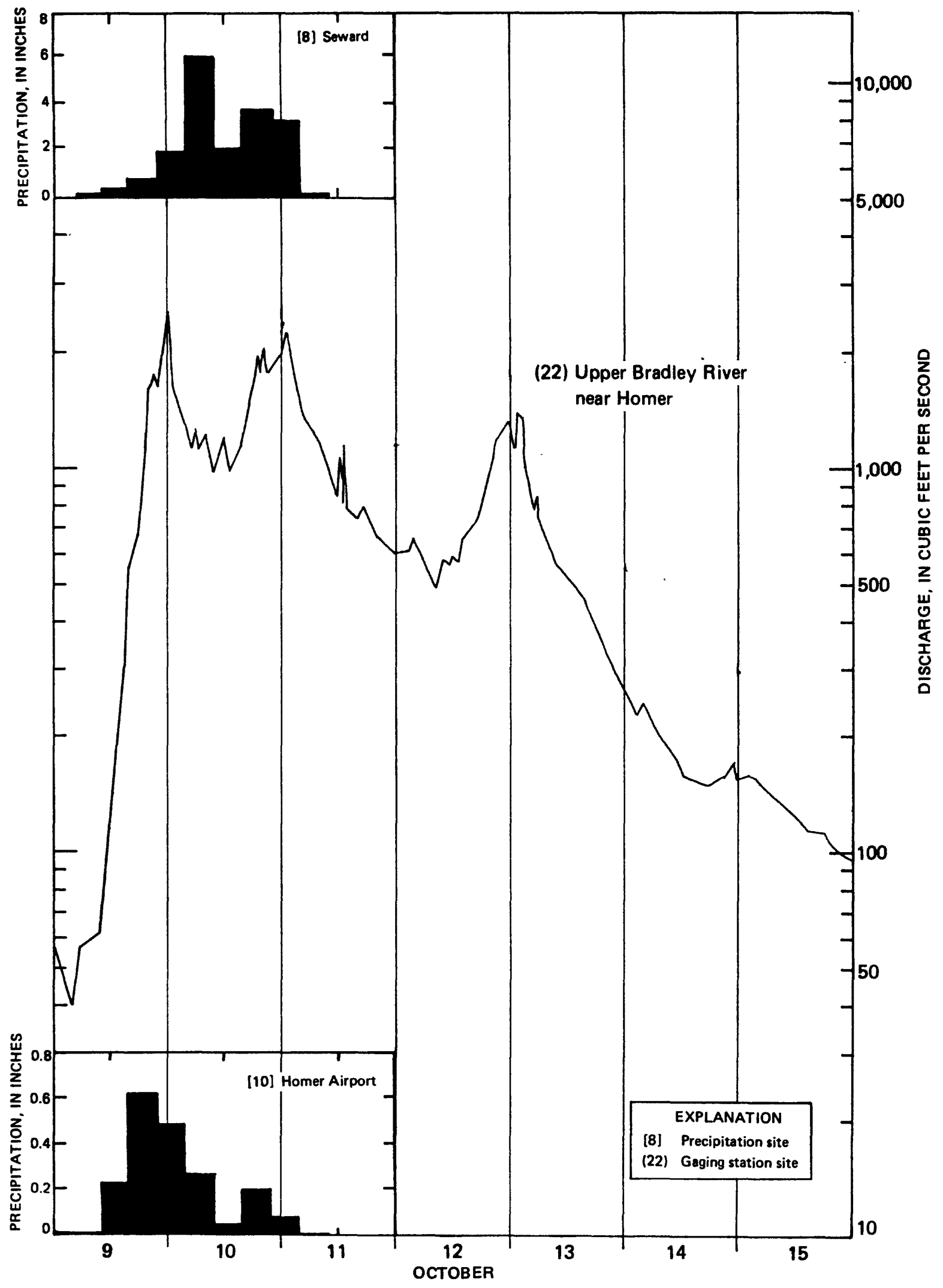

Figure 5.--Flood discharge hydrograph and concurrent 6-hour storm precipitation at selected sites in Kenai Peninsula. See table 3 for discharge data. 
River at Anchor Point (28), had recurrence intervals of 5 and 25 years, respectively. There was some minor erosion along Anchor River, which had much higher peak discharges on November 29 and 30,1983 . North of Homer, the maximum peak flow of record occurred at Beaver Creek near Kenai (39) and had a 20-year recurrence interval. Peaks at the sites mentioned above occurred October 10 , except at Beaver Creek, where the peak occurred on October 11. (See fig. 3B for accumulated precipitation at Kenai.)

\section{Seward Area Streams}

Within southcentral Alaska, the largest rainfall totals (fig. 2) and greatest rainfall intensity ( $f i g .3$ ) occurred in the vicinity of Seward ( $f i g$. $6)$, where it caused widespread catastrophic flooding. Landslides in the steep mountains bordering Resurrection Bay, eroding and migrating channels, and the subsequent deposition of debris compounded the problems caused by flood inundation. Most of this discussion of Seward area flooding is based on information provided by the authors' U.S. Geological Survey colleagues (Jones and Zenone, 1988). The record-breaking rains at Seward (see fig. 3 and earlier discussion in "Precipitation") caused widespread flooding near the mouth of Resurrection River and in streams tributary to Resurrection Bay. Peak discharge and stage at two Resurrection River sites, Exit Glacier bridge (9) and the discontinued gaging station at Seward (10), were only slightly higher than previously observed peak discharges. Neither of these sites has enough data to compute a recurrence interval.

The most severe damage and flooding were caused by streams tributary to Resurrection River near its mouth and by other small streams flowing into Resurrection Bay. Flood debris and alluvial sediment in the fast-moving streams clogged channels at bridges and culverts. This action resulted in overtopping and erosion of bridge approaches and railroad and highway embankments, interruption of highway and rail transportation, and damage or destruction of businesses and residences. The floodwaters caused noticeable erosion along the channels of several short, steep mountain streams and resulted in the subsequent deposition and channel migration in alluvial fans that have formed at the mountain front. Landslides on the steep mountain slopes bordering the headwaters of several streams deposited material in their channels and temporarily blocked streamflow. Surge releases of stored water and mass movement of earth, rock, vegetation, and water occurred when the temporary dam was overtopped and eroded; these surges produced peak discharges substantially in excess of previously observed flows. Measurement sites (6), (11), (13), and (19), at which the peak discharge was affected by storage releases are footnoted in table 2 .

The greatest peak discharge from the surge release of a debris dam occurred at Godwin Creek (6), where the discharge was estimated as $30,000 \mathrm{ft}^{3} / \mathrm{s}$. The unit runoff rate from the 13.8 square-mile basin was about $2,200\left(\mathrm{ft}^{3} / \mathrm{s}\right) / \mathrm{mi}^{2}$.

Boulders as large as $8 \mathrm{ft}$ in diameter were transported by the flow and subsequently deposited as the slope decreased near the mouth of Godwin Creek on Fourth of July Creek alluvial fan. The computed discharge could be too high because the channel at the survey site might have eroded after the peak stage occurred. 


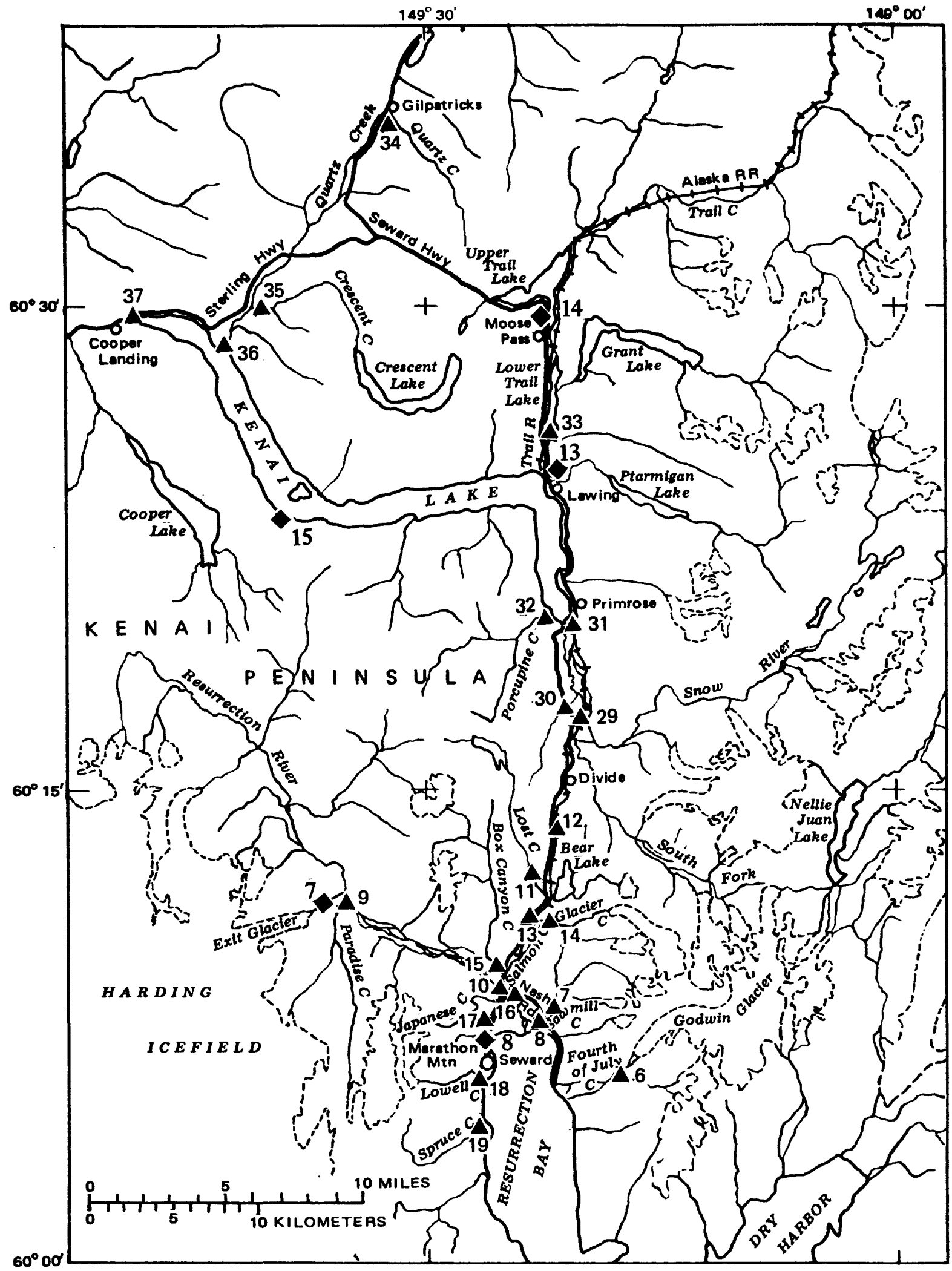

EXPLANATION

7 Rainfall measurement site . $\Delta 7$ Peak-discharge measurement site

Numbers refer to tables 1 and 2 respectively

Figure 6.--Rainfall measurement sites 7 to 8,13 to 15 and peak-discharge measurement sites 6 to 19, 29 to 37, Seward to Kenai Lake. 
Proceeding counter-clockwise around the head of Resurrection Bay, the next drainage basin is that of Sawmill Creek; flood discharge was determined at site (7), where the flow was confined, and downstream at Nash Road at site (8). The approximate discharge of $4,000 \mathrm{ft}^{3} / \mathrm{s}$ at Nash Road is the combined flow through the main bridge, No. 855, a secondary bridge, No. 854, and flow that bypassed both bridges and overtopped Nash Road.

The next major basin is Salmon Creek, which is tributary to Resurrection River just upstream from its mouth. Lost Creek is the largest contributor to Salmon Creek; Grouse Creek and Bear Creek are the largest Lost Creek tributaries. Bear Creek did not have a substantial flood peak because it drains Bear Lake, which was at a seasonal low-lake level. Discharges were determined at sites (11) through (16) in the Salmon Creek basin (table 2 and fig. 6). Salmon and Lost Creeks join just downstream from site (14) on Salmon Creek. The only site that has prior record is Lost Creek near Seward (11). The estimated peak discharge of Lost Creek, $14,000 \mathrm{ft}^{3} / \mathrm{s}$, was affected by the release of dammed water; recurrence intervals for this type of flood cannot be determined by standard flood-frequency analysis. Grouse Creek washed out small sections of the Seward Highway in a 1 -mile distance. Downstream from Lost Creek, floating debris and logs in Salmon Creek jammed in the pilings of the Alaska Railroad bridge, mile 6.0, and the pilings were sheared. Railroad bridges downstream, at miles 4.8 and 3.7 , were blocked and several hundred feet of track and embankment were washed out; extensive residential flooding occurred and subdivision roads were washed out. The peak flow of Salmon Creek at Nash Road (16) (10,300 $\left.\mathrm{ft}^{3} / \mathrm{s}\right)$, includes inflow from Box Canyon Creek, which was diverted during the flood into Clear Creek (15) - - peak discharge, 2,800 $\mathrm{ft}^{3} / \mathrm{s} \mathrm{-} \mathrm{and} \mathrm{which} \mathrm{then}$ entered Salmon Creek. The total flow past Nash Road included about 4,000 $\mathrm{ft}^{3} / \mathrm{s}$ of flow through the bridge, about $6,000 \mathrm{ft}^{3} / \mathrm{s}$ that overflowed the road, and bypass flow of about $300 \mathrm{ft}^{3} / \mathrm{s}$ that washed out Nash Road farther east.

Box Canyon Creek flood flows were not entirely diverted into Clear Creek; some of the remainder entered Resurrection River in a channel flowing south beside the Seward Highway embankment, inundating residential and commercial property. Still farther upstream along Resurrection River and Exit Glacier Road on the north bank, high flows in the steep mountain tributaries (including Box Canyon (reek) resulted in washouts, road overflow, and clogged bridges. The peak discharge, $19,000 \mathrm{ft}^{3} / \mathrm{s}$, at Resurrection River at Seward (10) is the total flow through the three highway bridges upstream of where Salmon Creek enters. The combined flood flows of Resurrection River and Salmon Creek caused extensive damage to residential and commercial property.

Several streams in Seward originate on the steep slopes of Mount Marathon. Japanese Creek, immediately south of Resurrection River, has a history of flooding along its alluvial fan. The flooding is often compounded by landslides and debris flows. Flood-fighting efforts by seward bulldozer operators on October 10 and 11 contained the creek within its most recent trench at the apex of the fan. The next stream to the south is not named on topographic maps, but is called Rudolph Creek (17) in table 2 of this report. The peak discharge of $1,020 \mathrm{ft}^{3} / \mathrm{s}$ for $1 \mathrm{mi}^{2}$ was the highest unit runoff measured in the vicinity of Seward from a basin that did not have a landslide dam upstream. Flood flows in Rudolph Creek and from several small tributaries washed out city streets and culverts, deposited coarse sediment in a lagoon and the small boat harbor, and eroded new channels along the base of Japanese Creek fan. 
The business district and most of the residential district of the "original" city of Seward are on the Lowell Creek alluvial fan. A diversion dam and tunnel built in 1940 to divert flood flows away from town and southward into Resurrection Bay prevented extensive flood damage in October 1986. Discharge through the tunnel during the recent flood cannot be determined, but the flood came within $0.7 \mathrm{ft}$ of overtopping the diversion dam. (The water surface of a flood in 1966 was $2 \mathrm{ft}$ below the crest of the diversion dam; see site (18) in table 2.)

In the Seward area, Spruce Creek (19) has the only continuous long-term (20 years) flood record. During the October 1986 storm, a massive debris avalanche occurred just upstream from the gaging station site. An estimated 3 million $\mathrm{ft}^{3}$ of material was removed; the remaining scar on the steep northern slopes of Spruce Creek canyon is about $0.7 \mathrm{mi}$ long and averages about $0.1 \mathrm{mi}$ wide. The avalanche material, including large trees, blocked the stream. When the temporary dam was breached, lateral bank and channel erosion resulted in the $0.3-\mathrm{mile}$ reach from the former dam to the head of Spruce Creek fan. Cross sections (fig. 7A) surveyed at the gage partially illustrate lateral scour on the north (left) bank. Farther downstream, flood flows on the alluvial fan were diverted northward by trees (from the avalanche area) deposited along the south channel bank, which prevented damage to the Sewage Treatment Plant. Inflow into the temporary catchment formed behind the avalanche debris dam was $5,420 \mathrm{ft} / \mathrm{f}^{3}$; the peak outflow of $13,600 \mathrm{ft}^{3} / \mathrm{s}$ includes the sudden release of water temporarily in storage before the debris dam failed.

\section{Kenai Lake Tributaries}

Flooding generally was less severe north of the divide on the Seward Highway between Seward and Kenai Lake. Peak discharges were determined at four sites, (29) to (32), on streams that flow into the upper end of the lake. An outstanding peak of $2,550 \mathrm{ft}^{3} / \mathrm{s}$ occurred at Sixteen Mile Creek (30); its headwaters lie between those of Grouse and Lost Creeks, which flow southward toward Seward. Unit runoff from Sixteen Mile Creek (30) was twice that in Grouse Creek (12) - 800 and $400\left(\mathrm{ft}^{3} / \mathrm{s}\right) / \mathrm{mi}^{2}$, respectively. Sixteen Mile Creek is a Snow River tributary, which enters between the gaging station, site (29), and site (31) at the two Seward Highway bridges across Snow River at the head of Kenai Lake. Peaks on Snow River are usually caused by outbursts from a glacierdammed lake in the headwaters. The last glacier-dammed breakout peak was December 2, 1985; the peak discharge was $12,000 \mathrm{ft}^{3} / \mathrm{s}$ at site (29). Maximum recorded discharge at this site was the August 31, 1967 outburst peak of 55,000 $\mathrm{ft}^{3} / \mathrm{s}$. The October 11,1986 peak, $17,500 \mathrm{ft}^{3} / \mathrm{s}$, was from storm runoff and was not from an outburst flood. Peak flow in snow River decreased in the intervening area between the gage and the highway bridges.

A crest-stage gage has been operated at Porcupine Creek near Primrose (32) since 1963. The peak of record, 4,000 $\mathrm{ft}^{3} / \mathrm{s}$, occurred on October 11, 1986. The stage-discharge relation changed because of changes in the channel cross section, lateral scour, and channel fill during the flood (fig. 7B). The first cross section shown in figure $7 B$ is just downstream from the apex of an alluvial fan, which has formed where the steep upstream-channel slope decreased as the stream approached its mouth; the second cross section is $420 \mathrm{ft}$ downstream and just upstream from Kenai Lake. Normally, during extreme floods, the peak stage of Kenai Lake occurs after the peak on Porcupine Creek and backwater occurs at the crest-stage gage. 

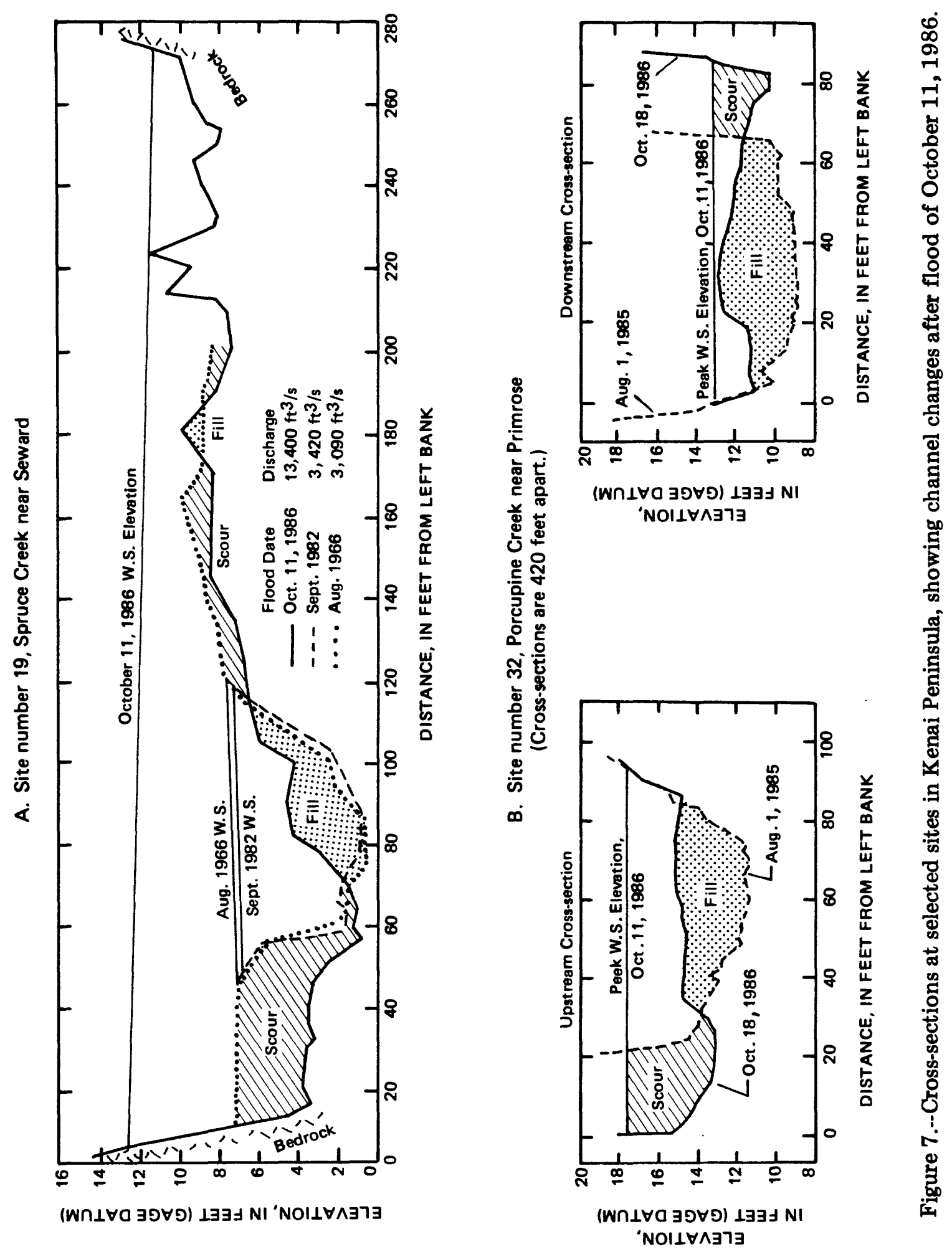
During the 1986 flood, peak stages and discharges were reduced by lake storage in streams with lakes upstream, because lake levels prior to the storm period were at a seasonal low. The Trail River near Lawing (33) gage essentially measured the stage of lake levels in Lower Trail Lake; Upper Trail and Grant Lakes provided additional upstream storage. The nearby precipitation gage at Lawing [13] had a 3-day total of 8.10 in. for October 9-11 (table 1), $8.00 \mathrm{in}$. of which fell between $4 \mathrm{p} . \mathrm{m}$. on October 9 and $4 \mathrm{a} . \mathrm{m}$. on 0 ctober 11 (Nibler, 1986) (fig. 3B). Stage at Trail River (33) rose from $4.20 \mathrm{ft}$ (discharge about $300 \mathrm{ft}^{3} / \mathrm{s}$ ) at $4 \mathrm{p} . \mathrm{m}$. October 9 to $9.0 \mathrm{ft}$ at 9 a.m. October 11 and to a maximum of $9.7 \mathrm{ft}$, from high-water marks, on the morning of October 12 (Nibler, 1986). Peak discharge was about 4,800 $\mathrm{ft}^{3} / \mathrm{s}$, based on the prior stagedischarge relation at this discontinued U.S. Geological Survey gaging station. Farther north, the discontinued crest-stage gage site Quartz Creek at Gilpatricks (34) had its highest known discharge in 25 years of observation since 1962. The effect of lake storage on peak discharges can be demonstrated by comparing the recurrence intervals at Trail River to those at Porcupine and Quartz Creeks; which were 5, 80, and 50 years, respectively.

Going downstream along Kenai Lake and River, precipitation generally decreased (fig. 2 and table 1). The effects of lake storage on the Kenai River peaks are shown by the delayed October 14 peak at Cooper Landing (37), which only had a 4-year recurrence interval, and by the October 18 peak at Soldotna (38), which had less than a 2-year recurrence interval.

\section{Anchorage}

The storm did not significantly affect the Anchorage area. The measurement sites in the vicinity of Anchorage, at which peak discharge and precipitation data were obtained, are shown in figure 8 . The recurrence intervals of the seven sites, (4) and (40) to (45), for which peak discharge data are shown ranged from less than 2 years to 15 years.

Flood hydrograph data and discharge tables (table 4) were compiled for two sites, Campbell Creek near Spenard (42) and East Fork Eklutna Creek near Palmer (44). The October 11 peak discharge on Campbell Creek was the highest in 21 years of record; recurrence interval was about 7 years (S. H. Jones, U.S. Geological Survey, written commun., 1987), as determined from a recent analysis using flood records collected throughout the Campbell Creek basin. Concurrent 6 -hour rainfall amounts at Anchorage Airport [24] are shown with the discharge hydrograph (fig. 9).

The hydrograph at East Fork Eklutna Creek near Palmer (44) is included because the drainage basin is at a relatively high altitude (average basin altitude, about $3,900 \mathrm{ft}$ ); the Geological Survey operates a weather station at the gage site, which is at an altitude of approximately $1,100 \mathrm{ft}$ above sea level. Streamflow was at a seasonal low of $73 \mathrm{ft}^{3} / \mathrm{s}$ on October 9 . Nighttime temperatures were below freezing during the period of October 5-9. However, temperatures increased to the 40 to $50{ }^{\circ} \mathrm{F}$ range in the morning of October 9 and increased still further to about $60^{\circ} \mathrm{F}$ on 0 ctober 10 . The increased temperature and $0.25 \mathrm{in}$. of rain on the morning of October 10 caused streamflow to rise significantly to 400 to $500 \mathrm{ft}^{3} / \mathrm{s}$. Later rains and continued high temperatures resulted in a peak discharge of $1,500 \mathrm{ft}^{3} / \mathrm{s}$ at $5: 30 \mathrm{a} . \mathrm{m}$. October 11 . (The effect of high-altitude rainfall and accompanying high air temperature on runoff from the glacier area, 19 percent of the basin of East Fork Eklutna Creek, is not known.) 


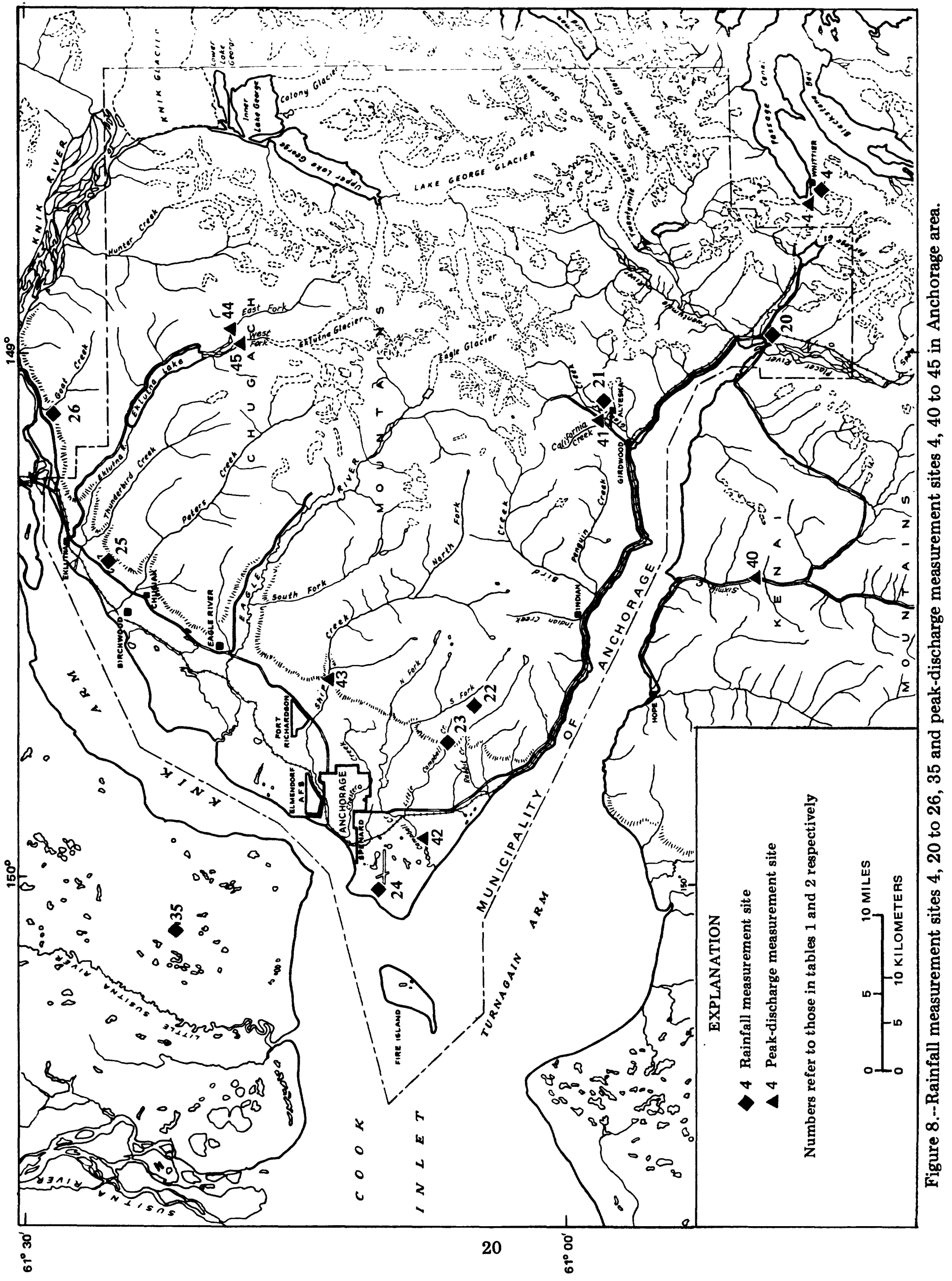




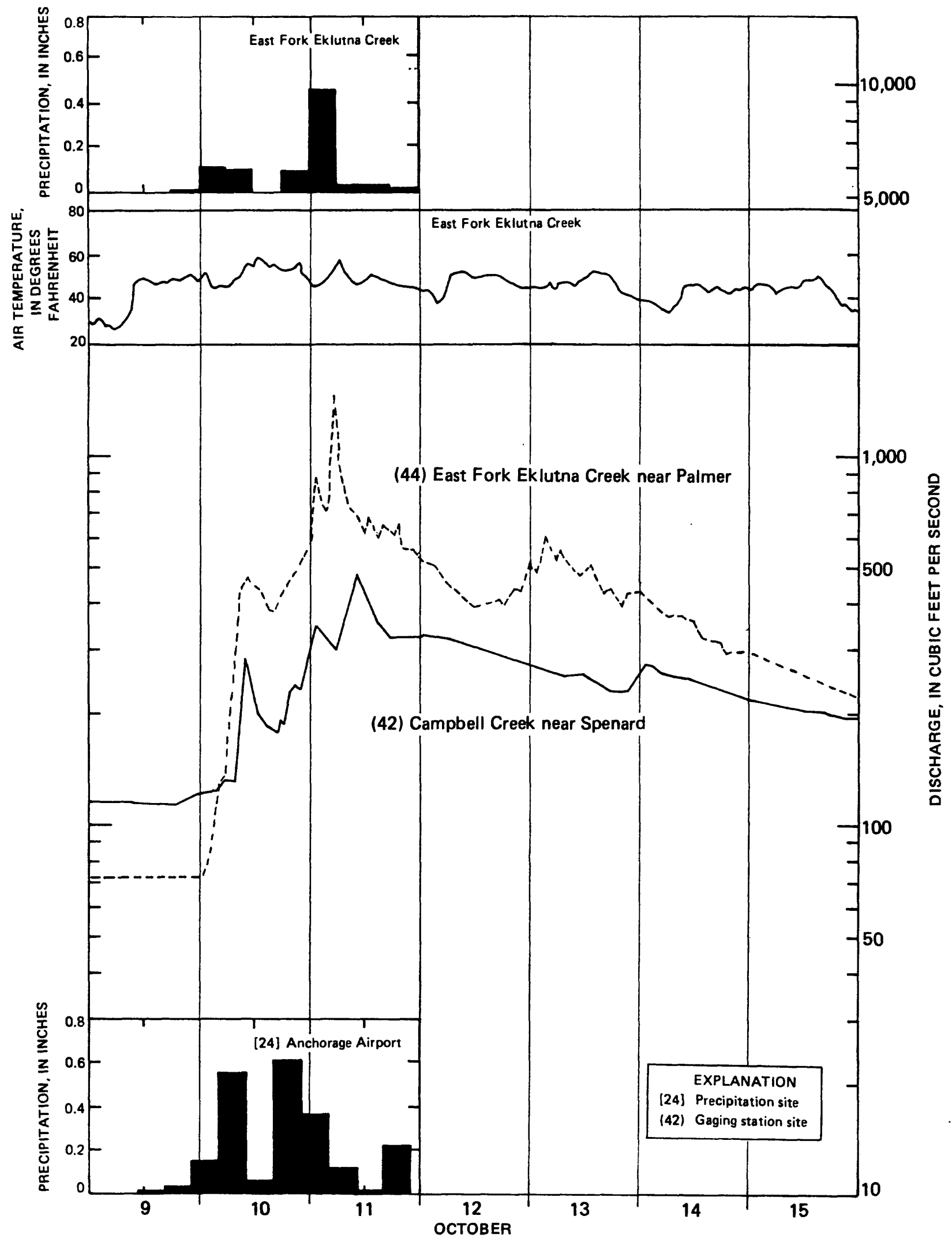

Figure 9.--Flood discharge hydrographs, concurrent 6-hour storm precipitation, and air temperature at selected sites in Anchorage area. See table 4 for discharge data. 
Table 4.--Discharge data for Campbell and East Fork Eklutna Creeks during October 1986

(42) 15274600 CAMPBELI CREEK MEAR SPENARD

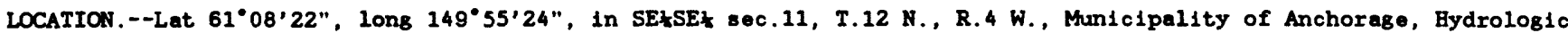
Unit 19050002 , on right bank $400 \mathrm{ft}$ upstream from bridge at Dimond Boulevard, $2.1 \mathrm{ml}$ upstream from mouth, and $4.3 \mathrm{ml}$ south of Spenard.

DRAINAGE AREA. $--69.7 \mathrm{mi}^{2}$.

GAGE-HEIGHT RECORD. - Water-stage recorder graph. Datum of gage is $1.68 \mathrm{ft}$ above Nat1onal Geodetic Vertical Datum of 1929. Prior to May 9, 1986, at site $400 \mathrm{ft}$ domstream at datum $16.36 \mathrm{ft}$ higher.

DISCBARGE RECORD.--Stage-discharge relation defined by current-meter measurements to $400 \mathrm{ft}^{3} / \mathrm{s}$.

MAXIMUM FOR PRIOR FLOOD RECORD.--(Water years 1966-86), $451 \mathrm{ft}^{3} / \mathrm{s}$, Aug. 13, 1981, gage he1ght, $3.54 \mathrm{ft}, 81 \mathrm{te}$ and datum then in use; maximum gage height recorded at prior site, $5.28 \mathrm{ft}$, Apr. 20 , 1976, backwater from $1 \mathrm{ce}$.

MAXIMUM FOR OCTOBER FLOOD.-- $472 \mathrm{ft}^{3} / \mathrm{s}, 1100$ hours Oct. 11, gage height, $20.89 \mathrm{ft}$.

Mean discharge, in cublc feet per second, October 1986

\begin{tabular}{|c|c|c|c|c|c|c|c|c|c|c|c|}
\hline Day & $\begin{array}{c}\text { Dis- } \\
\text { charge }\end{array}$ & Day & $\begin{array}{c}\text { Dis- } \\
\text { charge }\end{array}$ & Day & $\begin{array}{c}\text { D1s- } \\
\text { charge }\end{array}$ & Day & $\begin{array}{c}\text { Dis- } \\
\text { charge }\end{array}$ & Day & $\begin{array}{c}\text { Dis- } \\
\text { charge }\end{array}$ & Day & $\begin{array}{l}\text { D1s- } \\
\text { charge }\end{array}$ \\
\hline 1 & 120 & 6 & 122 & 11 & 352 & 16 & 192 & 21 & 156 & 26 & 115 \\
\hline 2 & 121 & 7 & 125 & 12 & 302 & 17 & 172 & 22 & 145 & 27 & 120 \\
\hline 3 & 155 & 8 & 121 & 13 & 253 & 18 & 164 & 23 & 138 & 28 & 119 \\
\hline 4 & 168 & 9 & 114 & 14 & 245 & 18 & 165 & 24 & 131 & 29 & 112 \\
\hline 5 & 137 & 10 & 198 & 15 & 206 & 20 & 172 & 25 & 120 & 30 & 105 \\
\hline Total & 4,967 & Mean & 160 & Maxim & 352 & & & & & & 102 \\
\hline
\end{tabular}

Gage height, in feet: discharge, in cublc feet per second; at indicated date and time in 1986

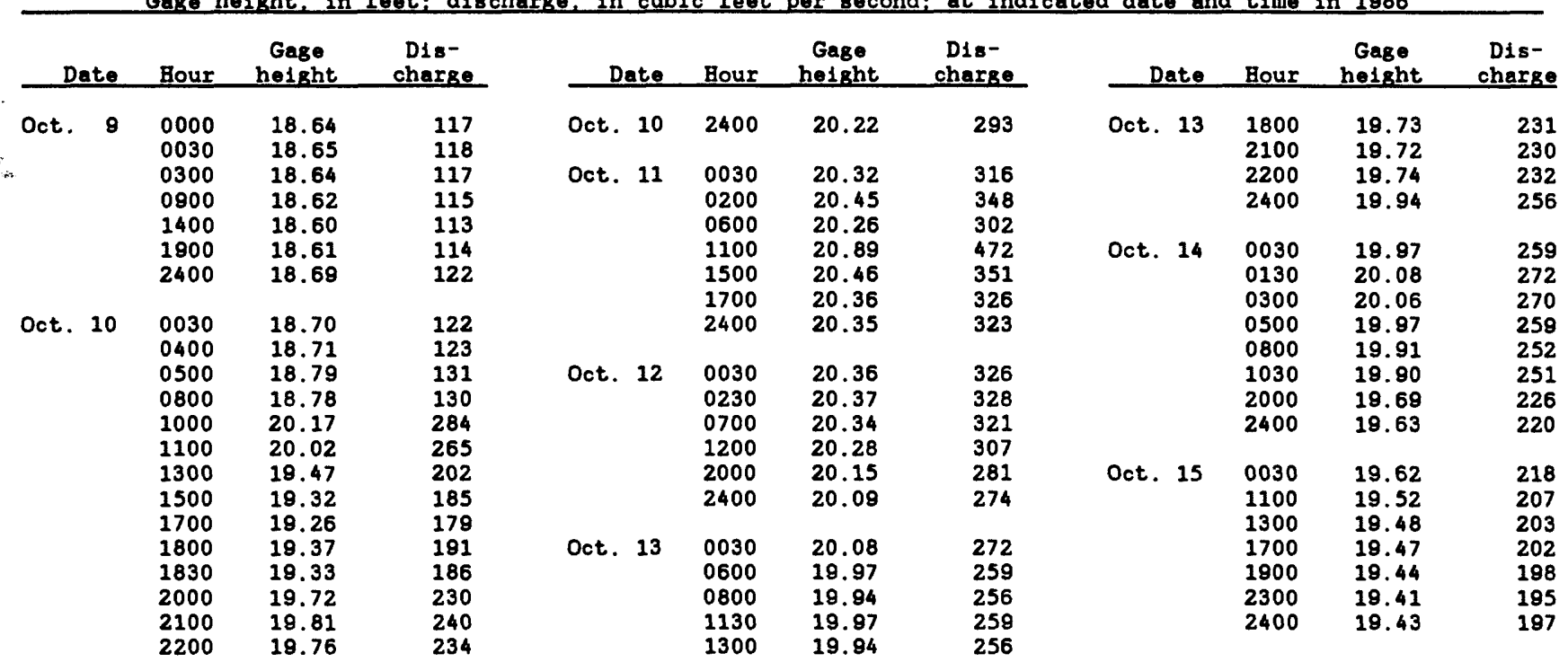


Table 4.--Discharge data for Campbell and East Fork Eklutna Creeks during October 1986-.Continued 15277600 EAST FORK EKLUTMA CREEK NEAR PALMER

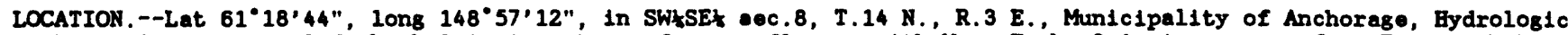
Unit 19050002 , on left bank $2.4 \mathrm{ml}$ upetream from confluence with Weot Fork, $3.1 \mathrm{ml}$ upetream from Ekiutna Lake and $20.5 \mathrm{mi}$ oouth of Palmer.

DRAIMAGE AREA. $--38.2 \mathrm{mi}^{2}$.

GAGE-HEIGBT RECORD.--Micrologger with 15-minute recording interval. Elovation of gage 101,080 ft above National Geodetic Vertical Datum of 1929, from topographic map. June 1960 to Sept. 1962 at 1 ite 0.2 mi downetream at different datum.

DISCBARGE RECORD.-- Stage-diecharge relation defined by current-meter meaourements below $600 \mathrm{ft} / \mathrm{s}$ and $\mathrm{slope-area}$ measurement at $1,500 \mathrm{et}^{3} / \mathrm{s}$.

MAXIMUM FOR PRIOR FLOOD RECORD.--(water years 1961-62, 85-86), 1, $320 \mathrm{ft}^{3} / \mathrm{s}$, Sopt. 12, 1961, gage height, $3.86 \mathrm{ft}$, site and datum then in use.

MAXIMUM FOR OCTOBER FLOOD.-- 1,500 $\mathrm{ft}^{3} / \mathrm{s}, 0530$ hours Oct. 11, gage helght, $9.19 \mathrm{ft}$.

\begin{tabular}{|c|c|c|c|c|c|c|c|c|c|c|c|}
\hline Day & $\begin{array}{c}\text { Dis- } \\
\text { charge }\end{array}$ & Day & $\begin{array}{c}\text { D1s- } \\
\text { charge }\end{array}$ & Day & $\begin{array}{c}\text { D1s- } \\
\text { charge }\end{array}$ & Day & $\begin{array}{c}\text { D1s- } \\
\text { charge }\end{array}$ & Day & $\begin{array}{c}\text { D1s- } \\
\text { charge }\end{array}$ & Day & $\begin{array}{c}\text { Dis- } \\
\text { charge }\end{array}$ \\
\hline $\begin{array}{l}1 \\
2 \\
3 \\
4 \\
5\end{array}$ & $\begin{array}{r}93 \\
92 \\
102 \\
110 \\
93\end{array}$ & $\begin{array}{r}6 \\
7 \\
8 \\
9 \\
10\end{array}$ & $\begin{array}{r}82 \\
79 \\
76 \\
73 \\
345\end{array}$ & $\begin{array}{l}11 \\
12 \\
13 \\
14 \\
15\end{array}$ & $\begin{array}{l}715 \\
445 \\
483 \\
350 \\
254\end{array}$ & $\begin{array}{l}16 \\
17 \\
18 \\
19 \\
20\end{array}$ & $\begin{array}{l}211 \\
180 \\
160 \\
142 \\
132\end{array}$ & $\begin{array}{l}21 \\
22 \\
23 \\
24 \\
25\end{array}$ & $\begin{array}{r}126 \\
115 \\
102 \\
94 \\
91\end{array}$ & $\begin{array}{l}26 \\
27 \\
28 \\
29 \\
30 \\
31\end{array}$ & $\begin{array}{l}84 \\
76 \\
71 \\
65 \\
61 \\
62\end{array}$ \\
\hline
\end{tabular}

Gage height. In fent: discharge. In cublc feat per second: at indleated date and time in 1986

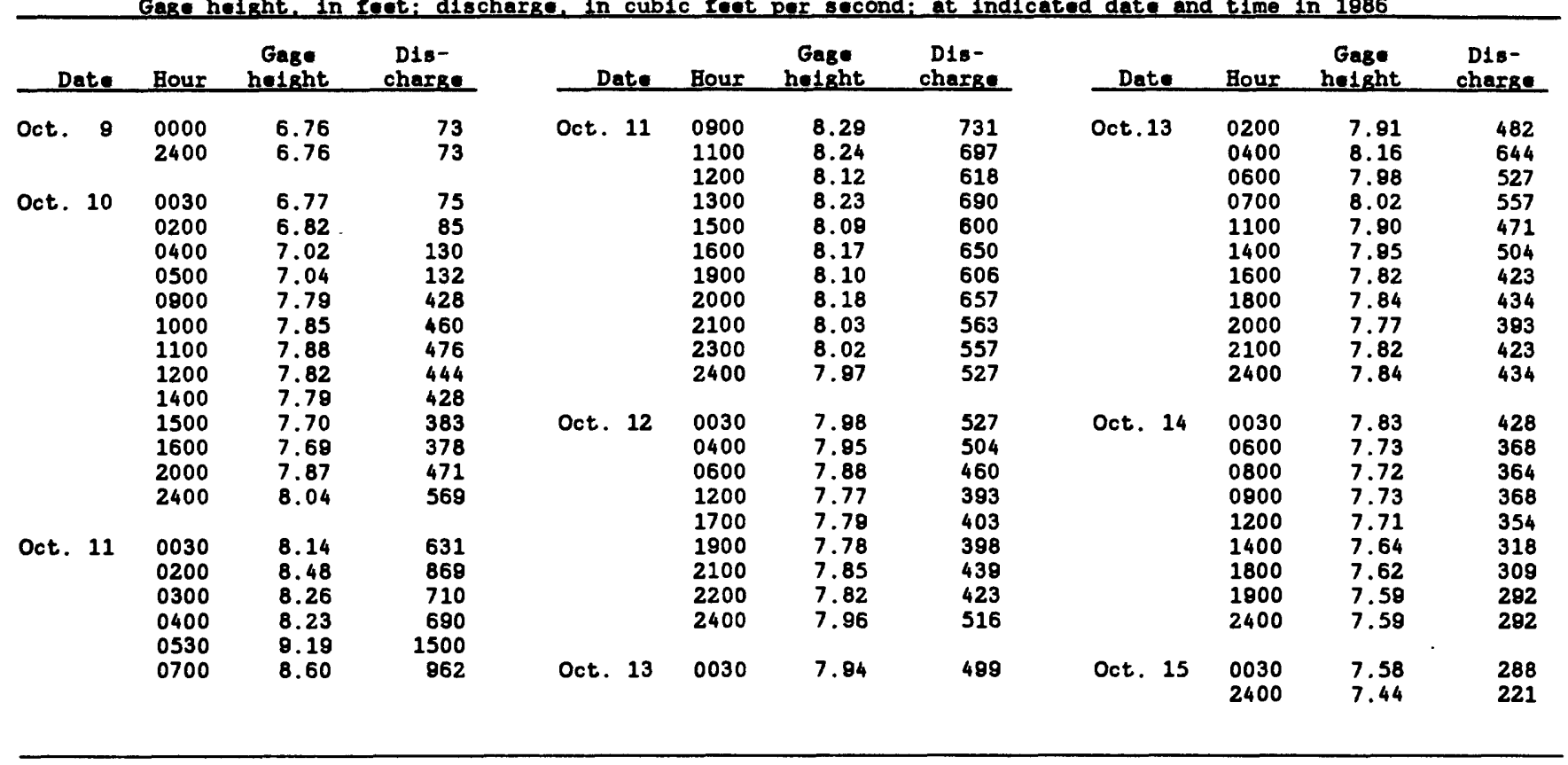




\section{Wasilla and Lower Susitna Valley}

The area affected by the flood is shown in figure 10. Some tributary streams to Knik Arm and Cook Inlet east of the Susitna River mouth had high flows. Recurrence intervals ranged from less than 2 years to about 3 years for the peak discharges of these streams. Rainfall increased going northwestward from Wasilla toward Willow and the recurrence intervals of peak discharges on Little Susitna River near Houston (50) and Deception Creek near Willow (63) were in the 10-to-15-year range.

The heaviest rainfall in the Susitna River basin occurred around Talkeetna (figs. 2 and 3C). Recurrence intervals of flood peaks increased from less than 2 years to almost 50 years moving downstream from Susitna River at Gold Creek (52), past Chulitna (53) and Talkeetna Rivers near Talkeetna (54), and to Susitna River at Sunshine (55). The discharge hydrograph of the flood for Talkeetna River (54) is plotted in figure 11 (values listed in table 5). The previous maximum discharge of record, on August 10, 1971, was less than the October 11, 1986 peak at the Talkeetna River gaging station. However, the 1971 peak at Susitna River at Sunshine (55) was more than the 1986 peak because the contribution from the upper Susitna River basin was larger during the 1971 flood (Lamke, 1972).

Severe flooding occurred in small streams west of Talkeetna. The 27square-mile drainage basin of Rabideux Creek near Sunshine (56) had a unit runoff of $100\left(\mathrm{ft}^{3} / \mathrm{s}\right) / \mathrm{mi}^{2}$. However, the unit runoff of $110\left(\mathrm{ft}^{3} / \mathrm{s}\right) / \mathrm{mi}^{2}$ at Moose Creek near Talkeetna (64) was the highest in the Susitna basin; recurrence interval of the Moose Creek peak discharge was 100 years. Southward from Talkeetna, flood damage along the Parks Highway was concentrated between Sunshine and Willow. The highway and railway bridges on Montana Creek were washed out and travel in the area was interrupted for a week. Peak discharge at Montana Creek near Montana (57) was 15,300 ft $3 / \mathrm{s}$; and the recurrence interval of the peak was determined to be greater than 100 years. The ratio of the maximum discharge in October 1986 to the peak discharge of a 100-year flood is 1.3 to 1 .

Flood flows from Goose Creek overtopped the Parks Highway. The combined peak flow of Goose Creek near Montana (58) and Sheep Creek near Willow (59) was $13,200 \mathrm{ft}^{3} / \mathrm{s}$; these two streams have intermingled flood flows. Since the 1971 flood, much of the flow from Sheep Creek has been diverted into Goose Creek, which has the smaller natural drainage area. The unit runoff of the combined flows and areas was $84\left(\mathrm{ft}^{3} / \mathrm{s}\right) / \mathrm{mi}^{2}$.

The recurrence interval of the October 1986 flood was 1.43 times the value of the discharge calculated for a 100-year flood at the long-term station, Caswell Creek (60), in operation since 1962. The unit runoff of $49\left(\mathrm{ft}^{3} / \mathrm{s}\right) / \mathrm{mi}^{2}$ was comparatively low for the area because the average basin altitude and channel slope are relatively low. Farther south, Little Willow Creek near Kashwitna (61) had considerable flow over the road. The October 1986 peak discharge was about 2.6 times the previous maximum discharge of the site's 8 years of record. Unit runoff on Little Willow Creek was $42(\mathrm{ft} / \mathrm{s}) / \mathrm{mi}^{2}$; recurrence interval is estimated at about 30 years. Unit runoff at Willow Creek near Willow (62) was $72\left(\mathrm{ft}^{3} / \mathrm{s}\right) / \mathrm{mi}^{2}$; peak recurrence interval was about 50 years. An 8 -mile stretch of unpaved highway between Willow and Hatcher Pass was severely damaged by Willow Creek, which flows beside the road embankment. Willow Creek's major tributary, Deception Creek (63), did not have the 


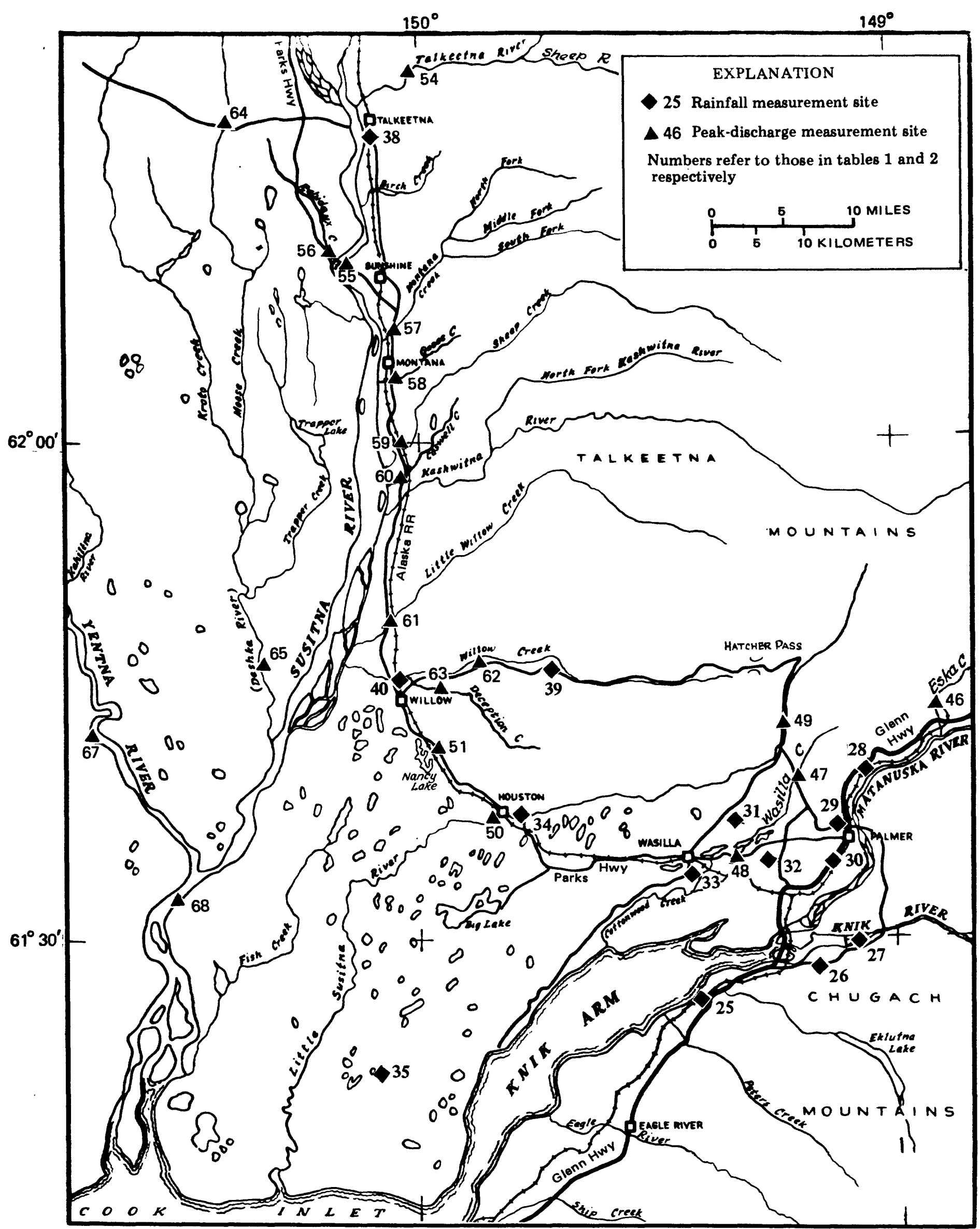

Figure 10.--Rainfall measurement sites 25 to 35,38 to 40 and peak-discharge measurement sites 46 to 51 , 54 to 65,67 to 68 in Wasilla and lower Susitna Valley area. 


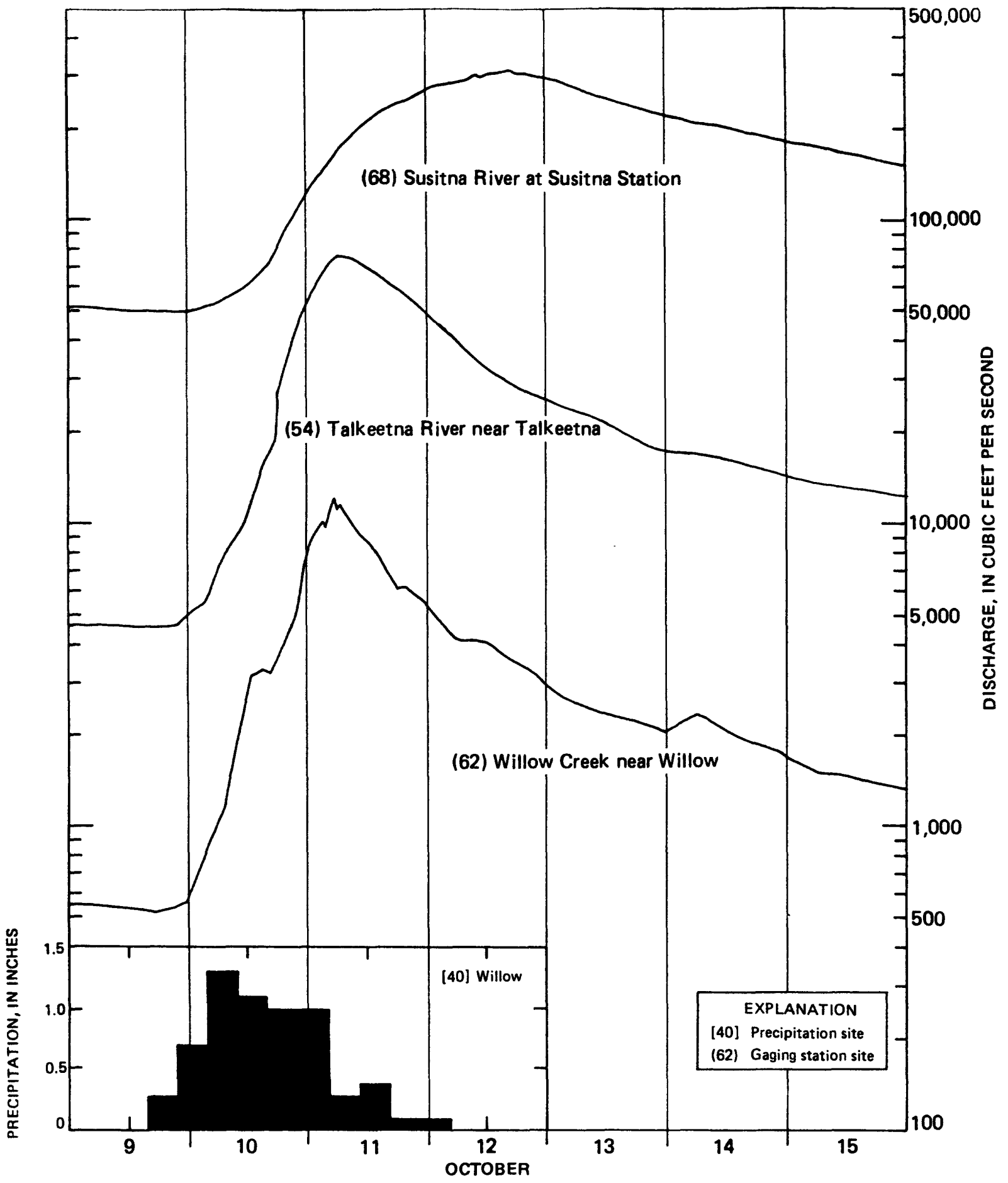

Figure 11.-Flood discharge hydrographs and concurrent 6-hour storm precipitation at selected sites in Susitna River basin. See table 5 for discharge data. 
Table 5.-Discharge data for three selected gaging stations in the lower Susitna River basin during October 1986 15292700 TALKEETNA RIVER NEAR TALKEETNA

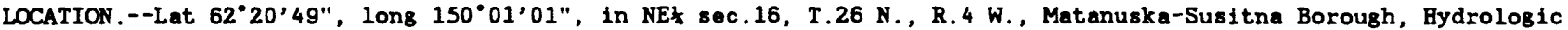
Unit 19050002, on left bank 1.7 mi downstream from Chunilna Creek, 3.5 mi northeast of Talkeetna, and about 5 mi above mouth.

DRAINAGE AREA. $--2,006 \mathrm{mi}^{2}$.

GAGE-HEIGHT RECORD.--Water-stage recorder sraph except Oct. 26-31. Elevation of sage 18 400 ft above National Geodetic Vertical Datum of 1929, from topographic map.

DISCHARGE RECORD.--Stage-discharge relation defined by current-meter measurements to 67,400 ft $3 / 8$. Discharge estimated Oct. 26-31.

MAXIMUM FOR PRIOR FLOOD RECORD.--(Water years 1964-86), 67,400 ft 3 /s, Aug. 10, 1971 , gage height, 16.35 ft.

MAXIMUM FOR OCTOBER FLOOD.-- 75,700 ft $3 / \mathrm{s}, 0600$ hours Oct. 11, gage height, $17.38 \mathrm{ft}$.

Mean discharge, in cubic feet per second. October 1986

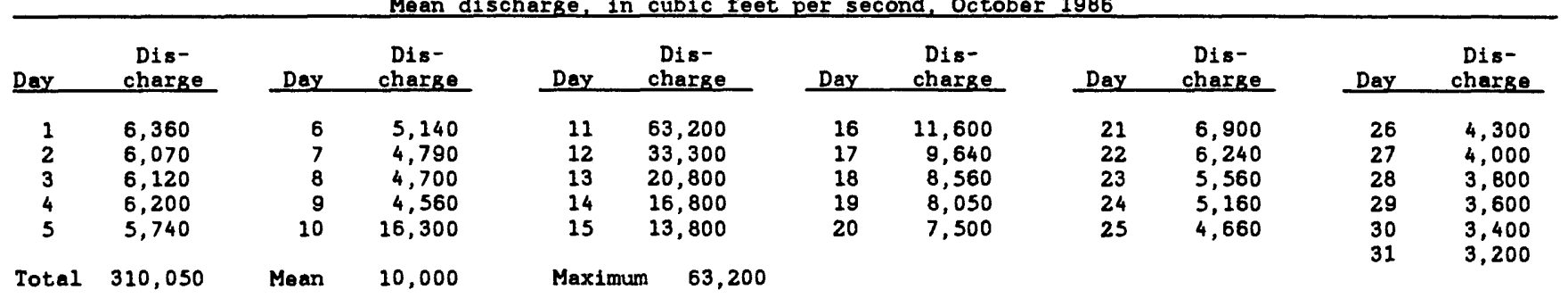

Gage height, in feet: discharge, in cubic feet per second: at indicated date and time in 1986

\begin{tabular}{|c|c|c|c|c|c|c|c|c|c|c|c|}
\hline Date & Hour & $\begin{array}{c}\text { Gage } \\
\text { height }\end{array}$ & $\begin{array}{l}\text { Dis- } \\
\text { charge }\end{array}$ & Date & Hour & $\begin{array}{c}\text { Gage } \\
\text { height }\end{array}$ & $\begin{array}{c}\text { Dis- } \\
\text { charge }\end{array}$ & Date & Hour & $\begin{array}{c}\text { Gage } \\
\text { height }\end{array}$ & $\begin{array}{c}\text { Dis- } \\
\text { charge }\end{array}$ \\
\hline Oct. $\quad 9$ & $\begin{array}{l}0000 \\
2000 \\
2230 \\
2400\end{array}$ & $\begin{array}{l}4.95 \\
4.92 \\
4.97 \\
5.07\end{array}$ & $\begin{array}{l}4,630 \\
4,560 \\
4,670 \\
4,900\end{array}$ & Oct. 11 & $\begin{array}{l}0130 \\
0230 \\
0330 \\
0430 \\
0600 \\
0630\end{array}$ & $\begin{array}{l}15.05 \\
15.65 \\
16.19 \\
16.65 \\
17.38 \\
17.20\end{array}$ & $\begin{array}{l}57,800 \\
62,000 \\
66,200 \\
69,900 \\
75,700 \\
74,200\end{array}$ & Oct. 13 & $\begin{array}{l}0500 \\
1000 \\
1330 \\
1800 \\
2400\end{array}$ & $\begin{array}{l}9.91 \\
9.58 \\
9.39 \\
9.16 \\
8.92\end{array}$ & $\begin{array}{l}22,900 \\
21,200 \\
20,200 \\
19,100 \\
18,000\end{array}$ \\
\hline Oct. 10 & $\begin{array}{l}0300 \\
0500 \\
0700 \\
0900 \\
1100 \\
1300 \\
1400 \\
1500 \\
1600 \\
1700\end{array}$ & $\begin{array}{l}5.35 \\
5.63 \\
5.95 \\
6.35 \\
6.75 \\
7.20 \\
7.55 \\
8.00 \\
8.50 \\
9.00\end{array}$ & $\begin{array}{r}5,530 \\
6,230 \\
7,070 \\
8,210 \\
9,520 \\
11,100 \\
12,400 \\
14,100 \\
16,200 \\
18,300\end{array}$ & & $\begin{array}{l}0630 \\
0730 \\
1100 \\
1400 \\
1700 \\
1900 \\
2100 \\
2300 \\
2400\end{array}$ & $\begin{array}{l}17.20 \\
17.33 \\
16.78 \\
16.20 \\
15.61 \\
15.03 \\
14.50 \\
14.03 \\
13.85\end{array}$ & $\begin{array}{l}74,200 \\
75,300 \\
70,500 \\
65,600 \\
60,800 \\
56,200 \\
52,000 \\
48,700 \\
47,300\end{array}$ & Oct. 14 & $\begin{array}{l}0200 \\
0700 \\
1000 \\
1200 \\
1400 \\
1600 \\
1830 \\
2400\end{array}$ & $\begin{array}{l}8.85 \\
8.74 \\
8.73 \\
8.73 \\
8.68 \\
8.60 \\
8.50 \\
8.34\end{array}$ & $\begin{array}{l}17,600 \\
17,200 \\
17,100 \\
17,100 \\
16,900 \\
16,500 \\
16,100 \\
15,400\end{array}$ \\
\hline & $\begin{array}{l}1800 \\
1900 \\
2000 \\
2100 \\
2200 \\
2300 \\
2400\end{array}$ & $\begin{array}{r}9.70 \\
10.40 \\
11.20 \\
12.00 \\
12.75 \\
13.50 \\
14.11\end{array}$ & $\begin{array}{l}22,000 \\
26,200 \\
31,000 \\
36,500 \\
42,400 \\
47,000 \\
51,300\end{array}$ & Oct. 12 & $\begin{array}{l}0130 \\
0330 \\
0600 \\
0830 \\
1200 \\
1500 \\
1900 \\
2400\end{array}$ & $\begin{array}{l}13.50 \\
13.00 \\
12.50 \\
12.00 \\
11.46 \\
11.07 \\
10.70 \\
10.31\end{array}$ & $\begin{array}{l}44,900 \\
41,500 \\
38,200 \\
35,000 \\
31,700 \\
29,400 \\
27,200 \\
25,000\end{array}$ & Oct. 15 & $\begin{array}{l}0600 \\
1200 \\
1700 \\
2000 \\
2200 \\
2400\end{array}$ & $\begin{array}{l}8.14 \\
7.93 \\
7.77 \\
7.69 \\
7.65 \\
7.62\end{array}$ & $\begin{array}{l}14,600 \\
13,700 \\
13,100 \\
12,800 \\
12,600 \\
12,500\end{array}$ \\
\hline
\end{tabular}


Table 5.--Discharge data for three selected gaging stations in the lower Susitna River basin during October 1986 -Continued 15294005 WILLOW CREEK TEAR WILLOW

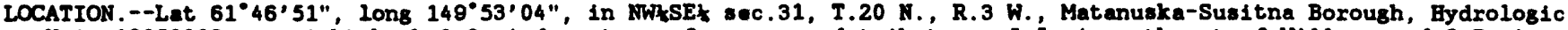
Unit 19050002 , on right bank $0.9 \mathrm{mi}$ domstream from unnamed tributary, 5.5 mi northeast of willow, and 6.7 mi upstream Irom Deception Creak.

DRAIKAGE AREA. $--166 \mathrm{mi}^{2}$.

GAGE-HEIGBT RECORD. --Water-stage recorder $8 \mathrm{raph}$ except for Oct. 31. Elovation of gage is $350 \mathrm{ft}$ above National Geodetic Datum of 1929, from topographic map. Prior to Apr. 2, 1981, at site 0.2 mi upstream at different datum.

DISCEARGE RECORD.-- Stage-discharge relation defined by current-meter measurements to $3,900 \mathrm{ft} / \mathrm{s}$ and extended to peek stage by indirect measurement.

MAXIMUM FOR PRIOR FLOOD RECORD.--(Water years 1978-1986), 4,450 ft $3 / 8$, July 28, 1980, gage height, 8.80 ft, at site and datum then in use.

MAXIMUM FOR OCTOBER FLOOD.-- $12,000 \mathrm{ft}^{3} / \mathrm{s}, 0530$ hours Oct. 11, gage height, $9.01 \mathrm{ft}$.

Mean discharge, in cubic leat per iecond, October 1986

\begin{tabular}{|c|c|c|c|c|c|c|c|c|c|c|c|}
\hline Day & $\begin{array}{c}\text { Dis- } \\
\text { charge }\end{array}$ & Day & $\begin{array}{c}\text { Dis- } \\
\text { charge }\end{array}$ & Day & $\begin{array}{c}\text { Dis- } \\
\text { charge }\end{array}$ & Day & $\begin{array}{c}\text { Dis- } \\
\text { charge }\end{array}$ & Day & $\begin{array}{c}\text { Dis- } \\
\text { charge }\end{array}$ & Day & $\begin{array}{c}\text { Dis- } \\
\text { charge }\end{array}$ \\
\hline $\begin{array}{l}1 \\
2 \\
3 \\
4 \\
5\end{array}$ & $\begin{array}{l}694 \\
731 \\
746 \\
516 \\
700\end{array}$ & $\begin{array}{r}6 \\
7 \\
8 \\
9 \\
10\end{array}$ & $\begin{array}{r}612 \\
565 \\
565 \\
548 \\
2,790\end{array}$ & $\begin{array}{l}11 \\
12 \\
13 \\
14 \\
15\end{array}$ & $\begin{array}{l}8,670 \\
4,020 \\
2,420 \\
2,040 \\
1,460\end{array}$ & $\begin{array}{l}16 \\
17 \\
18 \\
19 \\
20\end{array}$ & $\begin{array}{r}1,230 \\
1,010 \\
868 \\
789 \\
765\end{array}$ & $\begin{array}{l}21 \\
22 \\
23 \\
24 \\
25\end{array}$ & $\begin{array}{l}708 \\
654 \\
603 \\
572 \\
536\end{array}$ & $\begin{array}{l}26 \\
27 \\
28 \\
29 \\
30 \\
31\end{array}$ & $\begin{array}{l}466 \\
435 \\
361 \\
335 \\
318 \\
395\end{array}$ \\
\hline Total & 37,122 & Maan & 1,199 & Maximum & 8,670 & & & & & & \\
\hline
\end{tabular}

Gage heisht, in fent: discharge. in cubic feet per second; at indicated date and time in 1986

\begin{tabular}{|c|c|c|c|c|c|c|c|c|c|c|c|}
\hline Date & Houx & $\begin{array}{c}\text { Gage } \\
\text { heisht }\end{array}$ & $\begin{array}{c}\text { Dis- } \\
\text { charge }\end{array}$ & Date & Hour & $\begin{array}{c}\text { Gage } \\
\text { heisht }\end{array}$ & $\begin{array}{c}\text { Dis- } \\
\text { charge } \\
\end{array}$ & Date & Hour & $\begin{array}{c}\text { Gage } \\
\text { height }\end{array}$ & $\begin{array}{c}\text { Dis- } \\
\text { charge }\end{array}$ \\
\hline Oct. 10 & $\begin{array}{l}0000 \\
1700 \\
2000 \\
2400 \\
\\
0400 \\
0730 \\
1000\end{array}$ & $\begin{array}{l}3.47 \\
3.41 \\
3.44 \\
3.62 \\
\\
3.84 \\
4.23 \\
4.93\end{array}$ & $\begin{array}{r}558 \\
521 \\
539 \\
659 \\
\\
822 \\
1,160 \\
1,900\end{array}$ & Oct. 11 & $\begin{array}{l}0700 \\
0800 \\
1000 \\
1100 \\
1330 \\
1400 \\
1900 \\
2000\end{array}$ & $\begin{array}{l}8.77 \\
8.54 \\
7.98 \\
7.73 \\
7.48 \\
7.31 \\
6.46 \\
6.46\end{array}$ & $\begin{array}{r}11,400 \\
10,900 \\
9,580 \\
9,000 \\
8,420 \\
8,030 \\
6,100 \\
6,100\end{array}$ & Oct. 13 & $\begin{array}{l}0400 \\
0900 \\
1100 \\
1700 \\
2100 \\
2300 \\
2400\end{array}$ & $\begin{array}{l}4.87 \\
4.75 \\
4.68 \\
4.61 \\
4.53 \\
4.48 \\
4.47\end{array}$ & $\begin{array}{l}2,690 \\
2,480 \\
2,370 \\
2,260 \\
2,130 \\
2,060 \\
2,040\end{array}$ \\
\hline & $\begin{array}{l}1300 \\
1500 \\
1630 \\
2000 \\
2200 \\
2400\end{array}$ & $\begin{array}{l}5.81 \\
5.95 \\
5.97 \\
6.50 \\
6.96 \\
7.85\end{array}$ & $\begin{array}{l}3,070 \\
3,290 \\
3,320 \\
4,280 \\
5,280 \\
7,690\end{array}$ & Oct. 12 & $\begin{array}{l}2400 \\
0400 \\
0700 \\
0830 \\
1000 \\
1200\end{array}$ & $\begin{array}{l}6.16 \\
5.79 \\
5.58 \\
5.59 \\
5.59 \\
5.56\end{array}$ & $\begin{array}{l}5,430 \\
4,610 \\
4,150 \\
4,170 \\
4,170 \\
4,100\end{array}$ & Oct. 14 & $\begin{array}{l}0200 \\
0600 \\
0900 \\
1400 \\
1900 \\
2200 \\
2400\end{array}$ & $\begin{array}{l}4.51 \\
4.65 \\
4.60 \\
4.44 \\
4.33 \\
4.29 \\
4.21\end{array}$ & $\begin{array}{l}2,100 \\
2,320 \\
2,240 \\
2,000 \\
1,840 \\
1,790 \\
1,690\end{array}$ \\
\hline Oct. 11 & $\begin{array}{l}0200 \\
0300 \\
0330 \\
0530 \\
0600\end{array}$ & $\begin{array}{l}8.26 \\
8.51 \\
8.42 \\
8.01 \\
8.61\end{array}$ & $\begin{array}{r}9,050 \\
9,960 \\
9,630 \\
12,000 \\
11,100\end{array}$ & & $\begin{array}{l}1500 \\
1900 \\
2200 \\
2400\end{array}$ & $\begin{array}{l}5.40 \\
5.26 \\
5.12 \\
5.02\end{array}$ & $\begin{array}{l}3,750 \\
3,450 \\
3,160 \\
2,960\end{array}$ & Oct. 15 & $\begin{array}{l}0700 \\
1600 \\
2400\end{array}$ & $\begin{array}{l}4.06 \\
3.98 \\
3.89\end{array}$ & $\begin{array}{l}1,500 \\
1,410 \\
1,310\end{array}$ \\
\hline
\end{tabular}


Table 5.--Discharge data for three selected gaging stations in the lower Susitna River basin during October 1986 --Continued

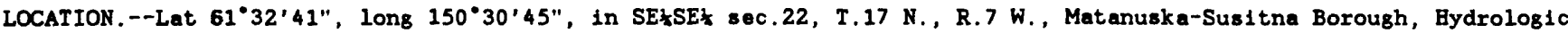
Unit 19050002 , on left bank at Susitna Station, $1.5 \mathrm{mi}$ downstream from Yentna River, and $12.5 \mathrm{mi}$ upstream from Alexander Creek.

DRAINAGE AREA. $--18,400 \mathrm{ml}^{2}$, approximetely.

GAGE-HEIGHT RECORD. --Water-stage recorder $8 \mathrm{raph}$ except Oct. 17-31, when the record was lost because of vandal1sm at the gaging station. Elevation of gage is $40 \mathrm{ft}$ above National Goodetic Vertical Datum of 1929, from topographic map.

DISCHARGE RECORD. --Stage-discharge relation defined by current-meter measurements to $221,000 \mathrm{ft} 3 / \mathrm{s}$. D1scharge for Oct. 17-31 estimated by comparison with nearby station record.

MAXIMUM FOR PRIOR FLOOD RECORD.--(Water years 1975-1986), 230,000 ft $3 / \mathrm{s}$, July 29, 1980 and Aug. 16, 1881; maximum gage height, $20.27 \mathrm{ft}$, Aug. 16, 1981.

MAXIMUM FOR OCTOBER FLOOD.-- $312,000 \mathrm{ft}^{3} / \mathrm{s}, 1700$ hours Oct. 12, gage he1ght, $22.58 \mathrm{ft}$.

Mean discharge, in cubic feet per second, October 1986

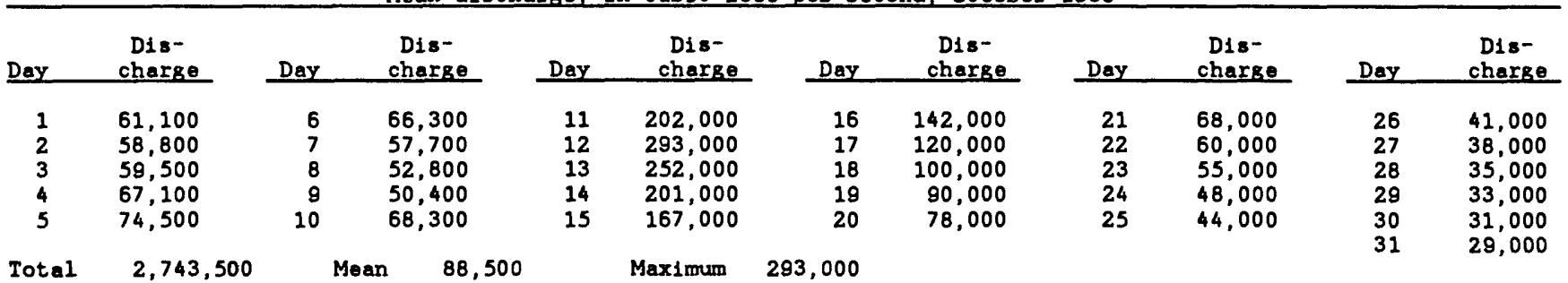

Gage height, in feet; discharge, in cuble feet per second; at indicated date and time in 1986

\begin{tabular}{|c|c|c|c|c|c|c|c|c|c|c|c|}
\hline Date & Bour & $\begin{array}{c}\text { Gage } \\
\text { helght }\end{array}$ & $\begin{array}{c}\text { Dis- } \\
\text { charge } \\
\end{array}$ & Date & Bour & $\begin{array}{c}\text { Gage } \\
\text { helght }\end{array}$ & $\begin{array}{c}\text { D1s- } \\
\text { charge }\end{array}$ & Date & Bour & $\begin{array}{c}\text { Gage } \\
\text { holght }\end{array}$ & $\begin{array}{c}\text { D1s- } \\
\text { charge }\end{array}$ \\
\hline Oct. 9 & $\begin{array}{l}0000 \\
0800 \\
1700 \\
2100 \\
2400\end{array}$ & $\begin{array}{l}9.83 \\
9.74 \\
9.68 \\
9.69 \\
9.73\end{array}$ & $\begin{array}{l}51,300 \\
50,500 \\
49,900 \\
50,000 \\
50,400\end{array}$ & Oct.11 & $\begin{array}{l}1230 \\
1500 \\
1700 \\
1900 \\
2200 \\
2400\end{array}$ & $\begin{array}{l}19.00 \\
19.64 \\
20.00 \\
20.25 \\
20.62 \\
20.86\end{array}$ & $\begin{array}{l}214,000 \\
230,000 \\
239,000 \\
246,000 \\
256,000 \\
263,000\end{array}$ & Oct. 13 & $\begin{array}{l}1100 \\
1400 \\
1800 \\
2100 \\
2400\end{array}$ & $\begin{array}{l}21.51 \\
20.13 \\
19.69 \\
19.45 \\
19.23\end{array}$ & $\begin{array}{l}253,000 \\
243,000 \\
231,000 \\
225,000 \\
220,000\end{array}$ \\
\hline Oct. 10 & $\begin{array}{l}0030 \\
0300 \\
0600 \\
0800 \\
1100 \\
1300 \\
1500 \\
1700 \\
1900 \\
2100 \\
2230\end{array}$ & $\begin{array}{r}9.74 \\
9.81 \\
9.93 \\
10.07 \\
10.37 \\
10.66 \\
11.06 \\
11.62 \\
12.38 \\
13.30 \\
13.95\end{array}$ & $\begin{array}{r}50,500 \\
51,200 \\
52,300 \\
53,900 \\
57,400 \\
61,200 \\
66,800 \\
73,900 \\
84,500 \\
98,900 \\
110,000\end{array}$ & Oct. 12 & $\begin{array}{l}0030 \\
0400 \\
0900 \\
1030 \\
1100 \\
1330 \\
1400 \\
1500 \\
1600 \\
1700\end{array}$ & $\begin{array}{l}20.91 \\
21.36 \\
21.92 \\
22.09 \\
22.02 \\
22.31 \\
22.23 \\
22.32 \\
22.45 \\
22.58\end{array}$ & $\begin{array}{l}264,000 \\
276,000 \\
293,000 \\
298,000 \\
296,000 \\
304,000 \\
302,000 \\
304,000 \\
308,000 \\
312,000\end{array}$ & Oct. 14 & $\begin{array}{l}0030 \\
0200 \\
0400 \\
0600 \\
0900 \\
1100 \\
1400 \\
1700 \\
2000 \\
2400\end{array}$ & $\begin{array}{l}19.20 \\
19.12 \\
18.95 \\
18.80 \\
18.64 \\
18.52 \\
18.30 \\
18.08 \\
17.88 \\
17.63\end{array}$ & $\begin{array}{l}219,000 \\
217,000 \\
213,000 \\
209,000 \\
206,000 \\
203,000 \\
198,000 \\
193,000 \\
188,000 \\
183,000\end{array}$ \\
\hline & 2400 & 14.61 & 121,000 & & $\begin{array}{l}1800 \\
2030\end{array}$ & & $\begin{array}{l}305,000 \\
302,000\end{array}$ & Oct. 15 & $\begin{array}{l}0030 \\
0300\end{array}$ & $\begin{array}{l}17.60 \\
17.43\end{array}$ & $\begin{array}{l}182,000 \\
178,000\end{array}$ \\
\hline Oct. 11 & $\begin{array}{l}0030 \\
0300 \\
0500 \\
0700 \\
0900 \\
1100\end{array}$ & $\begin{array}{l}14.80 \\
15.70 \\
16.37 \\
17.04 \\
17.73 \\
18.51\end{array}$ & $\begin{array}{l}124,000 \\
142,000 \\
155,000 \\
170,000 \\
185,000 \\
202,000\end{array}$ & Oct. 13 & $\begin{array}{l}2400 \\
0030 \\
0400 \\
0700\end{array}$ & $\begin{array}{l}21.98 \\
21.93 \\
21.47 \\
21.07\end{array}$ & $\begin{array}{l}294,000 \\
293,000 \\
280,000 \\
268,000\end{array}$ & & $\begin{array}{l}0400 \\
1000 \\
1400 \\
2100 \\
2400\end{array}$ & $\begin{array}{l}17.38 \\
17.03 \\
16.79 \\
16.45 \\
16.32\end{array}$ & $\begin{array}{l}177,000 \\
170,000 \\
164,000 \\
157,000 \\
154,000\end{array}$ \\
\hline
\end{tabular}


outstanding flows of the streams farther north. (See fig. 11 and table 5 for discharge hydrograph plot and data for Willow Creek.)

Moose Creek is a major tributary of the Deshka River. The gaging station on Deshka River near Willow (65) was washed out; overbank flood flows were spread out, slow-moving, and deep between the gage and $8 \mathrm{mi}$ downstream at the mouth. Peak discharge was $48,000 \mathrm{ft}^{3} / \mathrm{s}$. This discharge is about 1.3 times the estimated 100-year flood discharge. Skwentna River near Skwentna (66) had a peak stage of $17.3 \mathrm{ft}$, using highwater marks at the site (see fig. 1 for location). The stage-discharge relation in use when the station was discontinued, was used to determine that discharge was $69,000 \mathrm{ft}^{3} / \mathrm{s}$, which is higher than any peak discharge observed in 22 years of operation. A peak discharge of this size has a recurrence interval of 100 years. The maximum discharges at stations downstream near Susitna Station, Yentna (67) and Susitna Rivers (68), had recurrence intervals of about 10 and 40 years, respectively. The flood hydrograph of Susitna River at Susitna Station is shown in figure 11. (See table 5 for discharge data.)

Flooding also occurred in the small streams within the area between the Susitna River mouth and Tyonek (fig. 1). The precipitation gage at Beluga [43] had a total 3-day storm rainfall of 6.35 in. (table 1), most of which fell in the 24-hour period prior to $4 \mathrm{p.m}$. (ADST) on October 10 (U.S. Dept. of Commerce, 1986). Three discontinued stream-gaging stations west of the Susitna River mouth were checked for flood evidence. Two of the three sites had peak discharges higher than were experienced during their periods of operation. Channel changes at Capps Creek below North Capps Creek near Tyonek (69) were so extreme that a peak discharge value could not be determined. Based on the flood stage from high-water marks at the gage site and the prior stage-discharge rating at the gaging station, the discharge was estimated to have been greater than 1,200 $\mathrm{ft}^{3} / \mathrm{s}$. This is twice as high as any peak experienced in the 6 years of prior flood record. The recurrence interval of the October 10 flood at Chuitna River near Tyonek (70) probably was in the 50-to-100-year range. Unit runoff rates at both of these stations were near or greater than $100\left(\mathrm{ft}^{3} / \mathrm{s}\right) / \mathrm{mi}^{2}$. Chakachatna River near Tyonek, the other site that was checked, did not have any evidence of extraordinary flooding.

\section{Prince William Sound}

Precipitation ranged from 3.26 in. at Valdez [3] to 12.57 in. at Cordova North [2] (table 1). The only reported flood damage was locally in Cordova. Peak discharge at Power Creek near Cordova (1) (fig. 1) had a recurrence interval of 3 years. The recently re-established gage at Solomon Gulch near Valdez (3) experienced high flows. The maximum daily discharge of the October flood was almost as high as the maximum instantaneous peak of the 7 years of prior flood record. The stream (and Solomon Gulch Lake) are currently being used for a hydropower plant. A dam was constructed at the lake outlet to increase storage. The discharge over the flood spillway was determined at the gage on Solomon Gulch Bypass (2) and was 1.4 times as great as the previous instantaneous maximum. The streams around western Prince william Sound did not have especially high peaks. 
Federal Emergency Management Agency, 1986, Interagency hazard mitigation report: FEMA-782-DR-AK, Alaska, $40 \mathrm{p}$.

Jones, S.H., and Zenone, Chester, 1988, Flood of October 1986 at Seward, Alaska: U.S. Geological Survey Water-Resources Investigations Report 87-4278, 43 p.

Lamke, R.D., 1972, Floods of the summer of 1971 in South-Central Alaska: U.S. Geological Survey open-file report, $88 \mathrm{p}$.

1979, Flood characteristics of Alaska streams: U.S. Geological Survey Water-Resources Investigations 78-129, $61 \mathrm{p}$.

Nibler, G.J., 1986, Report on the October 10-12, 1986, heavy rain and flooding in Southcentral Alaska: Unpublished report on file at Alaska River Forecast Center in Anchorage office of National Weather Service, $15 \mathrm{p}$.

U.S. Department of Commerce, 1986, Climatological data, Alaska, October 1986: National Oceanic and Atmospheric Administration Monthly Report, v. 72, no. $10,30 \mathrm{p}$.

U.S. Weather Bureau, 1963, Probable maximum precipitation and rainfall-frequency data for Alaska: Washington, U.S. Weather Bureau Technical Paper 47, 69 p.

Water Resources Council, 1981, Guidelines for determining flood flow frequency: U.S. Water Resources Council Bulletin 17B, 195 p. 